\title{
Inequity averse optimization in operational research
}

\author{
Özlem Karsu ${ }^{\mathrm{a}, *}$, Alec Morton ${ }^{\mathrm{b}}$ \\ a Industrial Engineering Department, Bilkent University, Ankara, Turkey \\ ${ }^{\mathrm{b}}$ Management Science Department, University of Strathclyde Business School, Glasgow, UK
}

\section{A R T I C L E I N F O}

\section{Article history:}

Received 5 February 2014

Accepted 16 February 2015

Available online 20 February 2015

\section{Keywords:}

Inequity

Optimization

Multicriteria decision making

Equitable efficiency

fairness

\begin{abstract}
A B S T R A C T
There are many applications across a broad range of business problem domains in which equity is a concern and many well-known operational research (OR) problems such as knapsack, scheduling or assignment problems have been considered from an equity perspective. This shows that equity is both a technically interesting concept and a substantial practical concern. In this paper we review the operational research literature on inequity averse optimization. We focus on the cases where there is a tradeoff between efficiency and equity.

We discuss two equity related concerns, namely equitability and balance. Equitability concerns are distinguished from balance concerns depending on whether an underlying anonymity assumption holds. From a modeling point of view, we classify three main approaches to handle equitability concerns: the first approach is based on a Rawlsian principle. The second approach uses an explicit inequality index in the mathematical model. The third approach uses equitable aggregation functions that can represent the DM's preferences, which take into account both efficiency and equity concerns. We also discuss the two main approaches to handle balance: the first approach is based on imbalance indicators, which measure deviation from a reference balanced solution. The second approach is based on scaling the distributions such that balance concerns turn into equitability concerns in the resulting distributions and then one of the approaches to handle equitability concerns can be applied.

We briefly describe these approaches and provide a discussion of their advantages and disadvantages. We discuss future research directions focussing on decision support and robustness.
\end{abstract}

(C) 2015 Elsevier B.V. All rights reserved.

\section{Introduction}

There are various real life applications where equity concerns naturally arise and it is important to address these concerns for the proposed solutions to be applicable and acceptable. As a result, there exist many articles cited in the operational research (OR) literature that consider classical problems, such as location, scheduling or knapsack problems, and extend available models so as to accommodate equity concerns. These models are used across a broad range of applications including but not limited to airflow traffic management, resource allocation, workload allocation, disaster relief, emergency service facility location and public service provision. This broad range of applications indicates that considering these classical models with an emphasis on equity is practically relevant in addition to being technically interesting.

In this paper we present a literature review on inequity aversion in operational research and a classification of the modeling approaches

\footnotetext{
* Corresponding author. Tel.: +90 3122901960.

E-mail addresses: ozlemkarsu@bilkent.edu.tr(Ö. Karsu), alec.morton@strath.ac.uk (A. Morton).
}

used to incorporate concerns about equity alongside efficiency concerns in optimization problems. The equity concept is often studied in an allocation setting, where a resource or good is allocated to a set of entities. The concern for equity involves treating a set of entities in a "fair" manner in the allocation. The allocated resource or outcome can be a certain good, a bad or be a chance of a good or bad. The entities can be for example organizations, persons or groups of individuals which are at different locations or are members of different social classes.

At this point it may be helpful to look at three small examples. Let us start with a simple example in which we have two people who are allocated some money. Consider the following two allocations to these people, who are no different in terms of claim: $(100,50)$ and $(80,70)$. Common sense suggests that the second allocation is more equitable than the first one. The Pigou-Dalton principle of transfers (PD) formalizes this intuition. The PD states that any transfer from a poorer person to a richer person, other things remaining the same, should always lead to a less equitable allocation.

PD allows us to compare allocations that have the same aggregate amount as is the case in our simple example. However, things get more complicated when we have allocations that differ in terms 


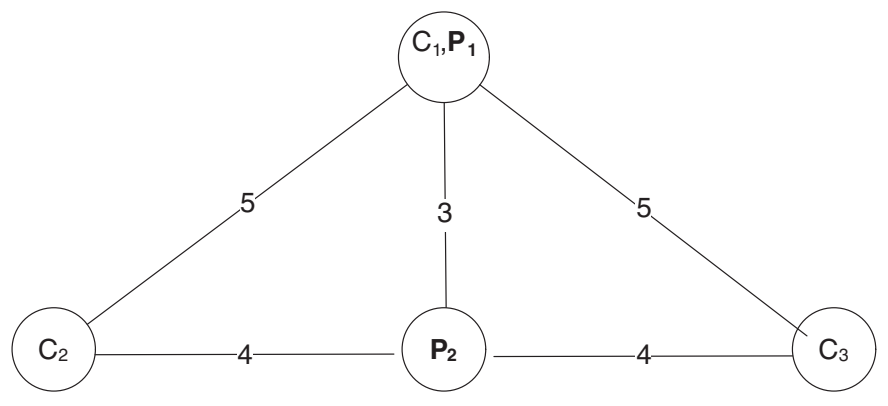

(b)

Fig. 1. Two alternative locations for an emergency service facility.

of the aggregate amount. In many situations an increase in equity results in a decrease in efficiency, which is usually measured by the total amount of the good (bad) that is allocated. As an example, consider a case where an emergency service facility is going be located. Suppose that a number of potential sites for the facility is already determined and the problem is to choose one of them. The facility will be serving different customers and it is important for the decision maker (DM) to ensure an equitable service to them. The DM evaluates how good a service is by the distance the customers have to travel to reach the facility: the shorter the distance between a customer and the facility, the better it is. One can consider choosing an alternative that minimizes the total distance that all the customers travel to the facility to evaluate how good each potential site is. However, in such a solution some of the customers may be significantly under-served. Fig. 1 shows a small example with 3 customers located at the nodes of a network $\left(C_{1}, C_{2}\right.$ and $\left.C_{3}\right)$. Suppose that there are two alternative locations for the emergency service facility $\left(P_{1}\right.$ and $P_{2}$, respectively). We will represent the two alternative locations using distance distributions that show the distance that each customer has to travel. The first location $\left(P_{1}\right)$ results in distance distribution $(0,5,5)$ and the second one $\left(P_{2}\right)$ results in distribution $(3,4,4)$. We see that the first alternative is more efficient in the sense that the total distance traveled is less. However, this efficiency is obtained at the expense of customers $C_{2}$ and $C_{3}$ who have to travel 5 units of distance. In the second alternative, the total distance traveled is larger but the distance traveled by the customers $C_{2}$ and $C_{3}$ is reduced. This is a typical example of the trade-off between efficiency and equity, which occurs in many real life situations. The DM's preferences would determine the better alternative in such cases: there is no "objective" way to determine which distribution is better, and reasonable people may take different views. For example the DM may argue that the first alternative is better claiming that it saves on total distance traveled, or s/he may argue that the second alternative is better as the maximum distance traveled is smaller. This review will focus on the cases where both efficiency and equity are of concern to the decision makers.

The above examples show cases where anonymity holds; that is, the identities of the entities are not important. However, as we will see in the next example, there may be situations where the entities have different characteristics and hence anonymity may not make sense. Suppose that you are the head of an academic department and you have to decide on the allocation of the next year's studentship budget to the Ph.D. students. Which of the following rules would you use as a base for your decisions?

- Allocate every student the same amount regardless of any other factor

- Allocate the budget proportional to the students' declared needs, which are measured as the shortfall from target income (students that need more get more)
Different people would give different answers to this question. The first rule respects person anonymity and hence is equitable. However, there are other sensible arguments that would favor other rules, as anonymity may be inappropriate when we have entities with different characteristics, such as different needs. These two rules involve two different dimensions of equity, "horizontal" and "vertical" equity. Horizontal equity is concerned with the extent to which entities within a class are treated similarly (Levinson, 2010); hence giving equal amounts to the students with the same need would satisfy concerns on horizontal equity. Vertical equity is concerned with the extent to which members of different classes are treated differently. Giving different amounts to students with different needs is a decision reflecting a concern for vertical equity.

As seen in this example, a reasonable equity concept might involve "unlike treatment of unlikes", such as giving different amounts to students with different needs. We call this equity concept that involves entities which are distinguished by an attribute such as need, claim or preferences balance.

\subsection{Review methodology}

The search methodology we use for this review is as follows: We used the "Web of Science" database for our search and used the keywords "equit*" (so that the words such as "equity" and "equitable" are included), "fairness" and "equality". We narrowed down the search by area (Operational Research/Management science) and we limited the search to "Journal Articles". As our focus is on current practice we surveyed the 10 years from 2003 to the time of analysis, mid way through 2013. For the "equit*" keyword, we have identified 392 articles. Screening by title, we eliminated the irrelevant ones, most of which use "equity" as a financial term, and obtained 181 articles. We further screened them by abstract. We focused on the studies that either report a modeling approach that incorporates equity concerns alongside efficiency concerns or discuss equity measures that have been used in the OR literature. We obtained 69 articles this way. For the "fairness" keyword we obtained 100 papers, which reduced to 34 after screening. As most of the articles found with the keyword "equality" use this term in its mathematical modeling sense (i.e. equality constraints in a mathematical model) only 4 articles obtained with this keyword were relevant. Scanning the references of these articles we added 27 articles to our review list.

Note that since our focus is inequity-averse optimization, we exclude the studies on non-cooperative games and filter these from the review. The articles on cooperative game theory concepts are also excluded as these concepts embody a stability rather than fairness rationale - they are solutions which can be made to "stick" rather than solutions which are attractive in an ethical sense. Moreover, we consider the approaches to problems where one has to trade equity off against efficiency and hence we do not review the solution approaches to the "fair division problem". We think there is a scope for another review for such problems. Note that if one does not have to trade equity off against efficiency, one does not have to answer the question "how much fairer is division A than division B?". It is enough to have ordinal information. In that sense, trading equity off against efficiency, brings an additional challenge to the allocation problems.

In Table 1 we report the journals that contribute to the literature with 3 or more publications. Around 14 percent of the articles were published in European Journal of Operational Research, followed by 10 percent and 8 percent in Computers and Operations Research and Operations Research, respectively. In total there were 43 journals, which shows that equity considerations arise in various settings and are discussed in publications in a variety of journals with different audiences and scopes.

The rest of the paper is as follows: Section 2 discusses the two main equity related terms, which are equitability and balance. We mention some of the applications involving equity concerns cited in 
Table 1

Number of articles by journal.

\begin{tabular}{ll}
\hline Journal & Frequency \\
\hline European Journal of Operational Research & 19 \\
Computers and Operations Research & 13 \\
Operations Research & 10 \\
Transportation Science & 9 \\
Annals of Operations Research & 9 \\
Journal of the Operational Research Society & 6 \\
Interfaces & 5 \\
Transportation Research Part B & 4 \\
Networks & 4 \\
Omega & 4 \\
Transportation Research Part E & 3 \\
Management Science & 3 \\
IEEE Systems Journal & 3 \\
Expert Systems with Applications & 3 \\
Queueing Systems & 3 \\
\hline
\end{tabular}

the OR literature. For such problems, we summarize the motivation for equity, the outcome distribution used in assessing equity and the entities for which equity is sought. In this section we do not attempt to give technical details on how the equity concerns are incorporated into mathematical models; we rather want to show that there is a wide range of applications and that equity is regarded as an important concern in the modeling process. Section 3 includes a more detailed discussion of different approaches taken in the literature to incorporate equitability and balance concerns in mathematical models. We conclude the discussion in Section 4 , where we point out future research directions that would be interesting to explore.

\section{Equitability and balance}

In this section we discuss two equity related concepts, namely equitability and balance. Equitability is used for comparing allocations across a set of indistinguishable entities. Balance concerns occur when we allocate goods over entities with different needs, claims or preferences. In such situations, ensuring justice might require treating different entities differently. We discuss these concepts in an order based on the frequency of appearance in our review.

\subsection{Equitability concerns}

Around two thirds of the articles in this review deal with equitability concerns. Equitability concerns occur when the set of entities are indistinguishable and hence anonymity holds. The first two examples used in the introduction show two important settings in which equitability can be a concern. The first setting is where a fixed amount of resource is being allocated and distributions can be quasi-ordered using PD. The second setting is where we have allocations with different total amounts which are not comparable using PD. This second setting makes things more interesting and complicated as there is often a tradeoff between efficiency and equitability. Hence this review focuses on such settings.

Earlier we gave an example regarding horizontal and vertical equity, which we relate to equitability and balance concepts, respectively. Alongside horizontal and vertical equity, equity can be quantified in other dimensions such as spatial equity and temporal equity (Levinson, 2010). Spatial equity is concerned with the extent to which the good is distributed equally over space, i.e. over the entities at different locations. Temporal equity, which is also referred to as longitudinal or generational equity, is the extent to which the good is distributed to the present or future recipients, i.e. to entities are distinguished by temporal aspects such as different generations who are the beneficiaries of a road investment or entities that use an emergency service system at different times.
Let us introduce some notation that will be used throughout the paper. Suppose that we have an outcome distribution (allocation) $y=\left(y_{1}, y_{2}, \ldots, y_{m}\right)$ where $y_{i}$ is the outcome level of entity $i \in I, I$ being the entity set. Without loss of generality, we assume that the more the outcome level, the better, i.e. the problem is a maximization problem. Note that it is possible to define the outcome distribution in multiple ways using different scales. For example, in a resource allocation problem two possible outcome definitions are the following: one can define the outcome distribution in terms of the absolute resource amounts allocated to different entities $\left(y_{i}\right)$ or as the shares of the total resource allocated to different entities $\left(y_{i} / \sum_{i \in I} y_{i}\right)$. An inequality index can be defined for either of the two distributions. The difference stems from the outcome definition rather than the index itself. In this work we do not distinguish the inequality indices based on how the distributions are scaled (see Marsh and Schilling (1994) for detailed information and a categorization of the inequality indices used in location theory).

We now provide a list of some of the many applications cited in the literature along with a discussion of the motivation for equity in such cases. We classify the applications based on the underlying technical problem.

\subsubsection{Allocation problems}

An equitable allocation of the good or resource over multiple entities is sought in such problems (Luss, 2012b). Applications include bandwidth or channel allocation (Tomaszewski, 2005; Lee et al., 2004; Lee \& Cho, 2007; Luss, 2008; Salles \& Barria, 2008; Ogryczak et al., 2008; Luss, 2010; Luss, 2012a; Jeong et al., 2005; Chang et al., 2006; Zukerman et al., 2008; Morell et al., 2008; Zhang \& Ansari, 2010; Bonald et al., 2006; Heikkinen, 2004; Ogryczak et al., 2005; Kunqi et al., 2007), water rights allocation (Udías et al., 2012), health care planning (Earnshaw et al., 2007; Demirci et al., 2012; Hooker \& Williams, 2012; Bertsimas et al., 2013), WIP (Kanban) allocation in production systems (Ryan \& Vorasayan, 2005), fixed cost allocation (Li et al., 2013; Butler \& Williams, 2006), and public resource allocation such as allocating voting machines to election precincts (Yang et al., 2013). There are also studies that consider general resource allocation settings such as Bertsimas et al. (2011), Bertsimas et al. (2012), Hooker (2010), Nace and Orlin (2007), Medernach and Sanlaville (2012) and Bertsimas et al. (2014).

One classical problem in this group is the discrete knapsack problem. The discrete knapsack problem selects a set of items such that the total value of the set is maximized subject to capacity constraints. In some applications equity is a concern as well as efficiency (total output maximization). A linear knapsack problem with profit and equity objectives is considered in Kozanidis (2009). Nace and Orlin (2007) introduce the lexicographically minimum and maximum load linear programming problems in order to achieve equitable resource allocations.

In resource allocation problems equity may be defined as spatial equity but other definitions are also possible such as space-time equity across members of the public in terms of the allocated amount. In water distribution problems, spatial and temporal equity across demand points is considered. One example of temporal equity concerns is averting high variation in water deficits in a region over multiple periods to avoid extreme deficits (Udías et al., 2012).

Bertsimas et al. (2011) discuss different fairness concepts that are used to ensure fair allocation of resources in an abstract environment. The authors derive bounds for the price of fairness, which is the loss in efficiency when a "fair" resource allocation is pursued. Bertsimas et al. (2012) also focus on balancing efficiency and equity in resource allocation settings. Bertsimas et al. (2014) propose a modeling framework for general dynamic resource allocation 
Table 2

Classical problems in OR re-considered with equity concerns.

\begin{tabular}{|c|c|}
\hline Problem & Examples \\
\hline Allocation & $\begin{array}{l}\text { Tomaszewski (2005), Lee, Moon, and Cho (2004), Lee and Cho (2007), Luss (2008), } \\
\text { Salles and Barria (2008), Ogryczak, Wierzbicki, and Milewski (2008), Luss (2010), Luss (2012a), } \\
\text { Jeong, Kim, and Lee (2005), Chang, Lee, and Kim (2006), Zukerman, Mammadov, Tan, Ouveysi, and Andrew (2008), Morell, Seco-Granados, } \\
\text { and Vázquez-Castro (2008), } \\
\text { Zhang and Ansari (2010), Bonald, Massoulié, Proutière, and Virtamo (2006), Heikkinen (2004), Ogryczak, Pioro, and Tomaszewski (2005), } \\
\text { Udías, Ríos Insua, Cano, and Fellag (2012), Earnshaw, Hicks, Richter, and Honeycutt (2007), Demirci, Schaefer, Romeijn, and Roberts (2012), } \\
\text { Hooker and Williams (2012), } \\
\text { Bertsimas, Farias, and Trichakis (2013), Ryan and Vorasayan (2005), Li, Yang, Chen, Dai, and Liang (2013), Butler and Williams (2006), } \\
\text { Yang, Allen, Fry, and Kelton (2013), Bertsimas, Farias, and Trichakis (2011), Bertsimas, Farias, and Trichakis (2012), Hooker (2010), } \\
\text { Nace and Orlin (2007), Medernach and Sanlaville (2012), Bertsimas, Gupta, and Lulli (2014), Karsu and Morton (2014), } \\
\text { Johnson, Turcotte, and Sullivan (2010), Kozanidis (2009), Eiselt and Marianov (2008), Vossen and Ball (2006), } \\
\text { Ball, Dahl, and Vossen (2009), Duran and Wolf-Yadlin (2011), Cook and Zhu (2005), Aringhieri (2009), } \\
\text { Wang, Fang, and Hipel (2007), Wang, Fang, and Hipel (2008), Swaminathan (2003), Swaminathan, Ashe, Duke, Maslin, and Wilde (2004), } \\
\text { Huang, Smilowitz, and Balcik (2012), Geng, Huh, and Nagarajan (2014), Kunqi, Lixin, and Shilou (2007) }\end{array}$ \\
\hline Location & $\begin{array}{l}\text { Batta, Lejeune, and Prasad (2014), Maliszewski, Kuby, and Horner (2012), Smith, Harper, and Potts (2013), Bell, Griffis, Cunningham, and } \\
\text { Eberlan (2011), } \\
\text { Ohsawa, Ozaki, and Plastria (2008), Chanta, Mayorga, and McLay (2011), Jia, Ordóñez, and Dessouky (2007), Melachrinoudis and } \\
\text { Xanthopulos (2003), } \\
\text { Ohsawa and Tamura (2003), Mladenovic, Labbe, and Hansen (2003), López-de-los Mozos, Puerto, and Rodríguez-Chía (2013), Lejeune and } \\
\text { Prasad (2013) } \\
\text { Mestre, Oliveira, and Barbosa-Póvoa (2012), Smith, Harper, Potts, and Thyle (2009), Ogryczak (2009), Berman, Drezner, Tamir, and } \\
\text { Wesolowsky (2009), } \\
\text { Baron, Berman, Krass, and Wang (2007), Suzuki and Drezner (2009), Galvão, Acosta Espejo, Boffey, and Yates (2006), Boffey, Mesa, Ortega, } \\
\text { and Rodrigues (2008), } \\
\text { Caballero, González, Guerrero, Molina, and Paralera (2007), Pelegrín-Pelegrín, Dorta-González, and Fernández-Hernández (2011), Johnson } \\
\text { (2003), Bashiri and Tabrizi (2010), } \\
\text { Marín, Nickel, and Velten (2010) }\end{array}$ \\
\hline Vehicle routing & $\begin{array}{l}\text { Beraldi, Ghiani, Musmanno, and Vocaturo (2010), Jang, Lim, Crowe, Raskin, and Perkins (2006), Blakeley, Bozkaya, Cao, Hall, and Knolmajer } \\
\text { (2003), Ramos and Oliveira (2011), } \\
\text { Campbell, Vandenbussche, and Hermann (2008), Vitoriano, Ortuño, Tirado, and Montero (2011), Huang et al. (2012), Perugia, Moccia, } \\
\text { Cordeau, and Laporte (2011), } \\
\text { Cappanera and Scutella (2005), Dell'Olmo, Gentili, and Scozzari (2005), Carotenuto, Giordani, and Ricciardelli (2007), Caramia, Giordani, } \\
\text { and Iovanella (2010) }\end{array}$ \\
\hline Transportation network design & $\begin{array}{l}\text { Azaiez and Al Sharif (2005), Stolletz and Brunner (2012), Tsai and Li (2009), Martin, Ouelhadj, Smet, Vanden Berghe, and Özcan (2013), } \\
\text { Turkcan, Zeng, Muthuraman, and Lawley (2011), Bollapragada and Garbiras (2004), Higgins and Postma (2004), Al-Yakoob and Sherali } \\
\text { (2006), } \\
\text { Balakrishnan and Chandran (2010), Erdogan, Erkut, Ingolfsson, and Laporte (2010), van 't Hof, Post, and Briskorn (2010), Briskorn and Drexl } \\
\text { (2009) } \\
\text { Kimbrel, Schieber, and Sviridenko (2006), Angel, Bampis, and Pascual (2008), Dugardin, Yalaoui, and Amodeo (2010), Smith et al. (2011) } \\
\text { Lo and Szeto (2009), Szeto and Lo (2006), Miyagawa (2009), Jahn, Möhring, Schulz, and Stier-Moses (2005), }\end{array}$ \\
\hline & Zhang and Shen (2010), Wu, Yin, Lawphongpanich, and Yang (2012) \\
\hline Other & $\begin{array}{l}\text { Bozkaya, Erkut, and Laporte (2003), Bergey, Ragsdale, and Hoskote (2003), Ogryczak and Śliwiński (2003), Mut and Wiecek (2011), } \\
\text { Kostreva, Ogryczak, and Wierzbicki (2004), Baatar and Wiecek (2006), Craveirinha, Girão Silva, and Clímaco (2008), Mclay and Mayorga } \\
\text { (2013), } \\
\text { Avi-Itzhak, Levy, and Raz (2008), Bonald et al. (2006), Mandelbaum, Momčilović, and Tseytlin (2012), Ward and Armony (2013), } \\
\text { Chan, Chung, and Wadhwa (2004), Sherali, Staats, and Trani (2003), Sherali, Staats, and Trani (2006), Sherali, Hill, McCrea, and Trani (2011), } \\
\text { Lulli and Odoni (2007), Barnhart, Bertsimas, Caramanis, and Fearing (2012), Tzeng, Cheng, and Huang (2007), Kotnyek and Richetta (2006), } \\
\text { Mukherjee and Hansen (2007), Ball, Hoffman, and Mukherjee (2010), Glover and Ball (2013), Davis, Samanlioglu, Qu, and Root (2013), } \\
\text { Armony and Ward (2010) }\end{array}$ \\
\hline
\end{tabular}

problems where there is a concern of equitably distributing the delay among the resource requests.

Another classical OR problem is the assignment problem which involves allocation of workload over agents. These problems may involve concerns on fairness among agents. Equity can be sought in terms of the assigned workload as in Eiselt and Marianov (2008). In air traffic management, when a foreseen reduction in a destination airport's landing capacity is anticipated, ground delay programs (GDP) are used as the primary tool for traffic flow management. In a GDP, the departure times of the affected flights are coordinated and hence the aircraft is delayed on ground. Vossen and Ball (2006) and Ball et al. (2009) model the GDP as an assignment problem and incorporate equity concerns.

We refer the interested reader to a recent article by Ogryczak, Luss, Pióro, Nace, and Tomaszewski (2014) for a comprehensive review of fair optimization models and methods in communication networks and location and allocation problems.

\subsubsection{Location problems}

One of the main concerns in facility location models is ensuring an equitable service to the population. Especially in essential public service facility location models, geographic equity of access to the service facilities is considered as one of the main requirements for an applicable solution. The access level can be measured in different terms such as the distance between demand points (customers) and the facilities (as in Batta et al., 2014; Maliszewski et al., 2012; Smith et al., 2013; Bell et al., 2011; Ohsawa et al., 2008; Chanta et al., 2011; Jia et al., 2007; Melachrinoudis \& Xanthopulos, 2003; Ohsawa \& Tamura, 2003; Mladenovic et al., 2003; López-de-los Mozos et al., 2013; Lejeune \& Prasad, 2013) or the time required to access the facility from the demand points as in Mestre et al. (2012) and Smith et al. (2009). Ogryczak (2009) considers the generic location problem from a multicriteria perspective and formulates a model where each individual access level is minimized (see Table 2). 
If the facilities are not essential service facilities, which can serve customers within a limited distance, the amount of population covered at each facility can be used as an indicator for which an equitable distribution is sought (Smith et al., 2013). A related problem is the equitable load problem, where ensuring an equitable service load distribution over the service facilities is of concern (Berman et al., 2009; Baron et al., 2007; Suzuki \& Drezner, 2009; Galvão et al., 2006).

Other problems include location-price setting problems, where equitable profit sharing between competing firms is addressed (Pelegrín-Pelegrín et al., 2011). Bashiri and Tabrizi (2010) consider the problem of locating warehouses and try to ensure equity in holding inventory among all supply chain members, because equity in inventory is argued to have a great impact on the future throughput of the company through competitiveness issues. Realizing that the solution which minimizes the total inventory often treats some retailers in an inequitable way, the authors seek equity across retailers in terms of the amount of inventory.

\subsubsection{Vehicle routing problems}

Vehicle routing problems are used in many applications such as pick-up and delivery service, disaster relief, hazardous material shipment and reverse logistics (e.g. waste collection).

One of the outcomes over which equity is sought in vehicle routing problems is vehicle workload (Jozefowiez, Semet, \& Talbi, 2008). In an effort to ensure an equitable workload distribution among vehicles in a multi vehicle pick-up and delivery problem, the expected length of the longest route is minimized in Beraldi et al. (2010). Similarly, Jang et al. (2006) consider a routing problem, and propose a model that guarantees that lottery sales representatives travel roads of similar length on different days. This ensures an equitable distribution of workload over a time period. Workload balance is also considered in Blakeley et al. (2003) in a periodic vehicle routing model used to optimize periodic maintenance operations. Ramos and Oliveira (2011) consider a reverse logistics network problem in which the service areas for multiple depots are defined. Equitable workload distribution to depots is considered in one of the objectives of their model. The workload of a depot is measured in terms of the hours needed to serve the service area it is assigned to.

Equity concerns naturally arise in vehicle routing problems considered in disaster relief contexts (Beamon \& Balcik, 2008). In such problems, one of the concerns of the decision makers is ensuring equitable service distribution to different affected areas (nodes). Equity of service to demand nodes is defined in various ways. For example, if all the demand is satisfied when a node is visited then the arrival time is used to measure service (Campbell et al., 2008).

Perugia et al. (2011) develop a multiobjective location-routing model, to model a home-to-work bus service, and try to achieve an equitable extra time distribution across customers. Extra time is defined as the difference between the bus transport time and the time of a direct trip from home to work.

\subsubsection{Scheduling}

In personnel scheduling, equitable systems attempt to distribute the workload fairly and evenly among employees (Ernst, Jiang, Krishnamoorthy, \& Sier, 2004). One of the popular problems in scheduling where equity plays a crucial role arises in healthcare organizations where nurses' or physicians' schedules are constructed (Azaiez \& Al Sharif, 2005; Stolletz \& Brunner, 2012; Tsai \& Li, 2009; Martin et al., 2013). In such settings providing an equitable distribution of workload across the nurses or physicians is important. The workload can be quantified in different terms such as the number of days on and off or in terms of the ratio of the nights shifts to day shifts.
In a class-faculty assignment problem, Al-Yakoob and Sherali (2006) seek equity in terms of the satisfaction (dissatisfaction) levels of the faculty members that have identical teaching loads. The dissatisfaction of a faculty member is measured by a function of the classes and time slots that the faculty member is assigned.

Fairness across patients is one of the factors considered while designing appointment systems (Cayirli \& Veral, 2003). For appointment scheduling for clinical services (Turkcan et al., 2011) introduce a model which includes equity related constraints in order to find uniform schedules for the patients assigned to different slots. The proposed unfairness measures are based on the expected waiting times at each slot and the number of patients in the system at the beginning of each slot.

Erdogan et al. (2010) propose bicriteria models to schedule ambulance crews, the two criteria being the aggregate expected coverage and the minimum expected coverage over every hour. The second criterion is included to incorporate temporal equity concerns into the model.

Sports scheduling is another problem where equity among competing teams is considered crucial (van 't Hof et al., 2010; Briskorn \& Drexl, 2009). One of the rules that is used to establish a certain degree of fairness in tournaments is ensuring that no team plays against the teams of the same strength group for a predetermined number of consecutive periods. The schedules that respect this rule are called group-balanced schedules (Briskorn \& Drexl, 2009).

Other examples include Kimbrel et al. (2006); Angel et al. (2008); and Dugardin et al. (2010). Kimbrel et al. (2006) deal with the problem of scheduling a multiprocessor, where fairness across (persistent) jobs in terms of the execution times is considered. Angel et al. (2008) consider equity in terms of the completion times of jobs in a setting where a set of $n$ jobs are to be processed by $m$ identical machines. They also consider the case where there is a concern of distributing the load, in terms of the processing (completion) time among the machines. Dugardin et al. (2010) consider reentrant hybrid flow shop scheduling problem, which allows the products to visit certain machines more than once. In this paper, the equity concept is used with a different underlying motive. The authors propose a bi-criteria model and use equity in order to generate solutions which are good enough in both criteria. That is, solutions that perform very well in one criterion while performing very badly in the other are avoided. This idea is explained in Section 3.

\subsubsection{Transportation network and supply chain design problems}

In transportation network design, equity over network users is considered (as in Lo and Szeto (2009), Szeto and Lo (2006), Miyagawa (2009), Jahn et al. (2005)).

Equity over users is considered while designing access control policies, in which meters are installed at on-ramps to control entry traffic flow rates. Different equity concepts are reported such as temporal equity and spatial equity: "The temporal equity measures the difference of travel time, delay and speed among users who travel on the same route but arrive at the ramp at different times while the spatial equity concerns the difference among users arriving at difference ramps at the same time" (Zhang \& Shen, 2010).

Equitable approaches are also used in congestion pricing schemes to ensure "fair" treatment of the travelers that are categorized for example by income or geographic locations (Wu et al., 2012; Levinson, 2010). Wu et al. (2012) consider a pricing scheme more equitable if it leads to a more uniform distribution of wealth across different groups of population.

Equitable capacity utilizations among the participating warehouses and manufacturers is considered in collaborative supply chain design (Chan et al., 2004). 


\subsubsection{Other integer/linear programming problems, combinatorial optimization problems and stochastic models}

In an effort to ensure equity over voters, in political districting problems the districts are desired to have approximately the same number of voters (referred to as "population equality") (Bozkaya et al., 2003). Bergey et al. (2003) study an Electrical Power Districting Problem, which deals with partitioning a physical grid into companies and incorporate equitable partitioning concerns across companies in terms of their earning potential.

Ogryczak and Śliwiński (2003), Ogryczak (2007), Mut and Wiecek (2011), Kostreva et al. (2004), Baatar and Wiecek (2006), approach equity from a multicriteria perspective and hence formulate multicriteria decision making models.

Craveirinha et al. (2008) consider a multiobjective routing optimization model in the context of MPLS (multiprotocol label switching) networks and consider equity in terms of the blocking probability of different services.

Markov decision process (MDP) models can also be considered with additional equity concerns. Mclay and Mayorga (2013) develop a linear programming (LP) model with side constraints on equity to model the dispatch of emergency medical servers to patients in an MDP framework. Different equity constraints are used to ensure both service and resource allocation equity over patients and workload and job satisfaction equity over servers.

In queuing systems one of the main concerns that have been recently discussed in the related literature is ensuring equity among the customers of the queuing system. Avi-Itzhak et al. (2008) define the fairness of the queue as "the fairness that can be related to the discipline or configuration of the queue when all customers are equally needy", that is the customers are identical in all respects except their arrival time and service requirements. One of the most popular queue disciplines First In, First Out (FIFO) takes arrival time (seniority) as its base when deciding who will be served next (the customer with the earliest arrival time is assigned to the server), while some other disciplines can be used that are centered on the service requirement factor (the customer with the shortest service requirement is assigned to the server) or on both of the seniority and service requirement factors. The authors discuss three measures that are used to quantify equity in queues in their paper. As the arrival time and/or the service requirement level of a customer are used as a basis for claim in the server allocation we discuss these measures in the balance section. Bonald et al. (2006) model a communication network problem, where the network is represented as a network of processor-sharing queues and analyze different fairness schemes.

There are also studies which mainly focus on equity over servers or (heterogeneous) server pools in queuing systems. One line of research on such systems deals with presenting and analyzing blind routing policies, i.e. policies of routing the customers to the server pools which require, at the time of decision, none or minimal information on the parameters of the system or the system state (based on Atar, Shaki, and Shwartz (2011), Mandelbaum et al. (2012)). Mandelbaum et al. (2012) propose such a blind policy that routes customers from emergency departments to hospital wards, which are modeled as heterogeneous server pools in a queuing system, where the servers are the beds. They consider equity over the ward staff in terms of two criteria: the first is the idleness ratios, the proportion of the idle servers in the server pools and the second is based on the flux ratios, i.e. the number of customers served by a server per time unit. Ward and Armony (2013) discuss a blind fair routing policy in large-scale service systems with customers and servers which are both heterogeneous. Equity is considered in terms of the server pool workloads, quantified using the their share of the server idleness (number of idle servers at each pool).

\subsection{Balance concerns}

About one third of the articles in our review deal with balance concerns. Balance is a special type of equity concern in which the entities are not necessarily treated anonymously since they differ in some equity-relevant characteristics such as needs, claims or preferences. Such problems do not have anonymity and an ideal solution may not give each entity the same proportion of the total allocation. See Kubiak (2009) (pages 5-6) for a discussion of applications in which proportional representation (in terms of resource allocation) according to these equity-relevant characteristics is one of the main concerns. Examples provided include ensuring that equal priority jobs with different lengths (or rights to resources) progress at the rates proportional to their lengths, or allocating bandwidths or processors according to the reciprocal of the packet size (the demand) of a customer in a network. "Evenly spread progress of tasks in time is necessary in such systems where the progress is proportional to the demand for the tasks's outcomes" (Kubiak, 2009). The author discusses such proportional representation problems from the optimization point of view also building upon the apportionment theory.

\subsubsection{Heterogeneity of needs (or size)}

The social equity concept quantifies equity based on the extent to which any good received is proportional to the need (Levinson, 2010).

As an example, Johnson et al. (2010) considers equity related concerns in a public policy problem faced by a municipality which has to select a portfolio of foreclosed homes to purchase to stabilize vulnerable neighborhoods. A spatial equity based objective is incorporated into the corresponding knapsack model, which minimizes the maximum disparity between the fraction of all purchased homes in a neighborhood and the number of available foreclosed houses in that neighborhood across all neighborhoods. In this example, the need of a neighborhood is quantified by the number of available foreclosed houses in that neighborhood.

In disaster relief settings the demand points have different needs. If partial satisfaction of demand is possible, the proportion of demand satisfied is used as a measure of service. Such measures are used by Davis et al. (2013) in an inventory management model and by Vitoriano et al. (2011) and Tzeng et al. (2007) in multi-objective transportation/distribution models. Davis et al. (2013) propose a stochastic programming model for placing commodities and distributing supplies in a humanitarian logistics network. There are studies that use more complicated service functions combining timing and proportion of demand satisfied (see e.g. Huang et al., 2012, which consider vehicle routing and supply allocation decisions in disaster relief). Similarly Swaminathan (2003) and Swaminathan et al. (2004) consider a drug allocation setting and provide each clinic with a fraction of drug supply which is proportional to their demand. Higgins and Postma (2004) propose an integer programming model to optimize siding rosters and ensure that growers with different amounts of cane maintain approximately the same percentage of cane harvested throughout the harvest season. Geng et al. (2014) consider a sequential resource allocation setting where each customer's utility is modeled as the ratio of the allocated amount to the demand.

In locating undesirable facilities such as waste disposal facilities, geographic equity in the distribution of nuisance effects or social rejection is one of the concerns that is incorporated into the models (Boffey et al., 2008; Caballero et al., 2007). In such problems the towns have different nuisance parameters since they have different sizes. A tenant-based subsidized housing problem is considered in Johnson (2003), where subsidy recipients are allocated to regions and equity across the potential host communities, which differ in size, has to be considered. 


\subsubsection{Heterogeneity of claims}

In some settings the entities are distinguishable based on their claims for a resource. The claims may be as a result of a previous legal agreement or on agreed upon rules. For example, in GDPs spreading delay or delay-related costs equitably among multiple airlines (flights or flight types) is one of the main concerns while assigning landing slots to airlines. In such settings the schedule which is generated before the disruptions is taken as a reference solution and hence may provide airlines with a basis to construct claims regarding the new schedule. For example a flight which was supposed to land first in the previous schedule would find it unfair if assigned as the last one in the new schedule.

Sherali et al. (2003), Sherali et al. (2006) develop an airspace planning and collaborative decision making model, which is a mixed integer programming model. The model is developed for a set of flights and selects a flight plan for each flight from a set of proposed plans. Each alternative plan consists of departure and arrival times, altitudes and trajectories for the flight. The suggested model addresses the equity issues among airline carriers in absorbing the costs due to rerouting, delays, and cancellations. Sherali et al. (2011) extend this model by integrating slot exchange mechanisms that allow airlines to exchange the assigned slots under a GDP. Lulli and Odoni (2007) propose an air traffic flow management model that assigns ground and air-borne delays to flights subject to both en route sector and airport constraints. The model is described as a macroscopic version of a previous model by Bertsimas and Stock Patterson (1998), with a different objective function, which is argued to "spread" the delay in an equitable way across affected flights. Similarly, Barnhart et al. (2012) propose integer programming models that are based on the models discussed in Bertsimas and Stock Patterson (1998) and Giovanni, Lorenzo, and Guglielmo (2000). The models assign ground holding delays to flights in a multiresource traffic flow environment that also take equity in delay distribution into account. By considering the en route sector capacity constraints, these models differ from the GDP models that only consider arrival airport capacity. Balakrishnan and Chandran (2010) consider the runway scheduling problem in airport transportation, which finds a schedule and corresponding arrival and departure times for aircraft. Equity among aircraft is ensured by the constraint position shifting approach. This approach requires that there is no significant deviation between positions of the aircraft in the optimized sequence and the first-come-first-served sequence. A similar approach is used in Smith et al. (2011). Ball et al. (2010) use a stochastic programming model that assigns ground delays to flights under uncertainty. The model minimizes expected delay and incorporates balance concerns among flights using a balance-related constraint.

Another application is scheduling commercials in broadcast television. Bollapragada and Garbiras (2004) propose a mathematical model for this problem, in which balance concerns over clients are also considered. Similarly, Karsu and Morton (2014) propose a bicriteria modeling framework that considers both efficiency and balance concerns in resource allocation problems.

\subsubsection{Heterogeneity of preferences}

In some problems entities have different preferences which make them distinguishable from each other. For example, Espejo, Marín, Puerto, and Rodríguez-Chía (2009) consider (as they call it) the minimum-envy location problem, where the customers have ordinal preference orderings for the candidate sites. The problem is opening a certain number of facilities to which the customers will be assigned. Each customer is assigned to his most preferred facility among those which are open and the envy between a pair of customers is measured as the difference between the ranks of the facilities.

\subsubsection{Diversity concerns}

Another concept which is related to equity but in an indirect or orthogonal way is diversity. Around 4 percent of the reviewed papers use the diversity concept. To see the motivation for this concept, suppose that you are going to select a set of candidates for a degree programme. You have concerns on diversity in the sense that you want certain population groups to have a certain degree of representation in the selected set. These groups may, for example, consist of people with a lower socioeconomic background. A common way of achieving this is to use quotas or proportion targets, i.e. ensuring that a certain proportion of the selected people will be from the specific group of concern. This approach involves treating people with different characteristics differently such that the selected team is diversified enough. For example, Bertsimas et al. (2013) ensure that the percentage distribution of (kidney) transplant recipients across different population groups are above specified lower bounds. Similarly, in an applicant selection model Duran and Wolf-Yadlin (2011) ensure diversity in the selected team in order to represent certain population groups.

Aringhieri (2009) considers the problem of forming teams of service personnel with different skills. To treat customers served by different teams equitably, the author introduces a diversity measure and ensures that the diversity is above a threshold for all the teams. To take another example of diversity, in hazardous material shipment, spreading risk over population groups in an equitable way is one of the main concerns (DellOlmo et al., 2005; Carotenuto et al., 2007; Caramia et al., 2010). In some studies the concept of equity of risk is handled by determining spatially dissimilar paths. These studies incorporate equity concerns by selecting a set of paths to carry the hazardous material, which are as dissimilar as possible. Two examples are due to DellOlmo et al. (2005) and Caramia et al. (2010), who consider the problem of selecting of $k$ routes in multiobjective hazardous material route planning. They use a measure of spatial dissimilarity and obtain an equitable distribution of risk over the related region by choosing spatially dissimilar paths to ship the hazardous material.

We do not devote a separate section to diversity and discuss it in this section under balance concerns. That is because although these studies address equity in a relatively indirect way, which is based on creating diversity, it is possible to conceptualize diversity as a balance concern in such settings. For example when selecting candidates for a degree program, the underlying problem can be considered as allocating admission to the degree program to population subgroups. Although there is no way in which degree admission can be allocated equally across people - out of $M$ people, only $m$ can be accepted onto the programme, and the remaining $M-m$ will have to be rejectedadmission can be allocated in a balanced way across the population subgroups by ensuring that the set of admitted candidates is diverse. Similarly, when selecting routes in hazardous material shipment settings, the membership of the selected route(s), i.e. being a node on the route, is allocated to different population centres. Diversity ensures an equitable allocation of membership over different nodes avoiding inequitable solutions such as a solution in which most of the routes pass through the same set of nodes exposing these nodes to much higher risk than the rest.

\section{Different approaches to handle equity concerns}

\subsection{Different approaches to incorporate equitability}

Equity has been widely discussed in the economics literature where it is generally accepted that there is no one-size-fits-all solution and that special methods are required to handle equity concerns in particular cases (see e.g. Sen, 1973 and Young, 1994, who discusses different concepts of equity). Nevertheless, using transparent and explicit rules that determine what is equitable and what is not or how 
Table 3

Solution approach framework.

\begin{tabular}{|c|c|c|}
\hline Approach & & Examples \\
\hline Rawlsian & & $\begin{array}{l}\text { Ohsawa and Tamura (2003), Melachrinoudis and Xanthopulos (2003), Mladenovic et al. (2003), Baron et al. (2007), } \\
\text { Davis et al. (2013), Campbell et al. (2008), Maliszewski et al. (2012), Pelegrín-Pelegrín et al. (2011), } \\
\text { Boffey et al. (2008), Bell et al. (2011), Berman et al. (2009), Mestre et al. (2012), } \\
\text { Johnson (2003), Caballero et al. (2007), Baron et al. (2007), Jia et al. (2007), } \\
\text { Tzeng et al. (2007), Perugia et al. (2011), Miyagawa (2009), Ryan and Vorasayan (2005), } \\
\text { Demirci et al. (2012), Udías et al. (2012), Johnson et al. (2010), Bashiri and Tabrizi (2010), } \\
\text { Beraldi et al. (2010), Prokopyev, Kong, and Martinez-Torres (2009), Earnshaw et al. (2007), Erdogan et al. (2010), } \\
\text { Bertsimas et al. (2011), Chanta et al. (2011), Yang et al. (2013), Mclay and Mayorga (2013), } \\
\text { Li et al. (2013), Batta et al. (2014), Vitoriano et al. (2011), Geng et al. (2014), } \\
\text { Martin et al. (2013), Zhang and Ansari (2010), Craveirinha et al. (2008), Heikkinen (2004), } \\
\text { Bertsimas et al. (2014), Angel et al. (2008), López-de-los Mozos et al. (2013), Butler and Williams (2006) }\end{array}$ \\
\hline Lexicographic extension & & $\begin{array}{l}\text { Vossen and Ball (2006), Luss (2010), Luss (2008), Nace and Orlin (2007), } \\
\text { Luss (2012a), Salles and Barria (2008), Wang et al. (2007), Wang et al. (2008), } \\
\text { Lee and Cho (2007), Lee et al. (2004), Tomaszewski (2005), Hooker (2010), } \\
\text { Ogryczak et al. (2005), Bonald et al. (2006), Medernach and Sanlaville (2012) }\end{array}$ \\
\hline Inequality index based & $\begin{array}{l}\text { Range } \\
\text { Mean deviation } \\
\text { Variance } \\
\text { Gini coefficient } \\
\text { Sum of pairwise deviations }\end{array}$ & $\begin{array}{l}\text { Boffey et al. (2008), Kozanidis (2009), Turkcan et al. (2011), Mclay and Mayorga (2013), } \\
\text { Stolletz and Brunner (2012), Martin et al. (2013), Kimbrel et al. (2006), Ramos and Oliveira (2011) } \\
\text { Ogryczak (2009), Eiselt and Marianov (2008), Martin et al. (2013), Bergey et al. (2003), } \\
\text { Bertsimas et al. (2014), López-de-los Mozos et al. (2013), Jang et al. (2006), Galvão et al. (2006) } \\
\text { Turkcan et al. (2011), Tsai and Li (2009), Chan et al. (2004), Blakeley et al. (2003) } \\
\text { Lejeune and Prasad (2013), Wu et al. (2012) } \\
\text { Ohsawa et al. (2008), Al-Yakoob and Sherali (2006), Lejeune and Prasad (2013) }\end{array}$ \\
\hline Aggregation function based & $\begin{array}{l}\text { Social welfare functions } \\
\text { Equitable efficiency }\end{array}$ & $\begin{array}{l}\text { Marín et al. (2010), López-de-los Mozos, Mesa, and Puerto (2008), Martin et al. (2013), Ball et al. (2009), } \\
\text { Hooker and Williams (2012), Bertsimas et al. (2012), Kunqi et al. (2007) } \\
\text { Kostreva et al. (2004), Ogryczak et al. (2008), Dugardin et al. (2010), } \\
\text { Mut and Wiecek (2011), Baatar and Wiecek (2006) }\end{array}$ \\
\hline
\end{tabular}

equitable a given distribution is on a cardinal or sometimes ordinal scale can be useful in ensuring that the decisions are applicable and acceptable.

Similarly, in operational research there are many different ways of incorporating equitability in the decision process since its precise interpretation depends on both the structure of the problem at hand and the decision maker's understanding of a "fair" distribution. In this section, we discuss the operational research approaches that incorporate equitability concerns in mathematical models alongside other concerns (mostly efficiency).

One of the most common and simplest ways to incorporate equitability concerns is focusing on the min ( $\max$ ) level of outcomes across persons. This approach is called the Rawlsian principle (Rawls, 1971). The Rawlsian principle is justified using a veil of ignorance concept, which assumes that the entities do not know what their positions (the worst-off, the second worst-off etc.) will be in the distribution. To illustrate, suppose that you are given two distributions over two people generically named A and B, such as $(5,50)$ and $(30,25)$. You have to choose one of the allocations and then will learn whether you are A or B. You would seriously consider choosing $(30,25)$ as you might be the worse-off person in a distribution and would get only 5 units if you choose $(5,50)$. This ignorance is a reason to consider the worst-off entities in the distribution as any entity should find the distribution acceptable after learning its position. This approach, however, fails to capture the difference between distributions that give the same amount to the worst-off entity: two distributions such as $(1,1,9)$ and $(1,5,5)$ are indistinguishable in terms of inequity from a Rawlsian point of view although the latter is significantly more equitable from a common sense point of view. This drawback can be avoided by using a lexicographic extension, which will be discussed later in detail.

A more sophisticated approach to incorporate equitability concerns would be using summary inequality measures in the model. We call such approaches inequality index based approaches. These approaches can be further categorized based on whether the index is employed in a constraint while defining the feasible region or is used as one of the criteria in the objective function.
A more general, and hence more complicated, approach would be to use a (inequity-averse) aggregation function and to maximize it. We refer to such approaches as aggregation function based approaches. Some studies optimize a specific function of the distribution and obtain a single equitable solution while others use a multi-criteria approach and obtain a set of equitable solutions.

The above classification is summarized in Table 3. We will discuss these approaches further in the following sections.

\subsubsection{The Rawlsian approach $\left(\min _{i} y_{i}\right)$}

These methods represent equity preference by focusing on the worst-off entity, hence the minimum outcome level in a distribution (Rawls, 1971). Some studies try to maximize the minimum outcome while others restrict it in a constraint that makes sure that it is above a predefined value. The studies encountered that use a Rawlsian approach to equitability are Ohsawa and Tamura (2003), Melachrinoudis and Xanthopulos (2003), Mladenovic et al. (2003), Baron et al. (2007), Davis et al. (2013), Campbell et al. (2008), Maliszewski et al. (2012), Pelegrín-Pelegrín et al. (2011), Boffey et al. (2008), Bell et al. (2011), Berman et al. (2009), Mestre et al. (2012), Johnson (2003), Caballero et al. (2007), Baron et al. (2007), Jia et al. (2007), Tzeng et al. (2007), Perugia et al. (2011), Miyagawa (2009), Ryan and Vorasayan (2005), Demirci et al. (2012), Udías et al. (2012), Johnson et al. (2010), Bashiri and Tabrizi (2010), Beraldi et al. (2010), Prokopyev et al. (2009), Earnshaw et al. (2007), Erdogan et al. (2010), Chanta et al. (2011), Bertsimas et al. (2011), Yang et al. (2013), Mclay and Mayorga (2013), Li et al. (2013), Batta et al. (2014), Geng et al. (2014), Martin et al. (2013), Zhang and Ansari (2010), Craveirinha et al. (2008), Heikkinen (2004), Bertsimas et al. (2014), Angel et al. (2008), López-de-los Mozos et al. (2013), Butler and Williams (2006). Clearly, this is an easy to implement and popular approach.

The Rawlsian approach is the one of the oldest approaches in OR used to incorporate a fairness concept into the models. Many classical OR problems such as assignment, scheduling and location have also been studied with "bottleneck" objectives. For example, the facility location problems that locate $p$ facilities such that the maximum distance between any demand point and its nearest facility is minimized are known as $p$-center problems. These models assign each demand 
point to its nearest facility, hence full coverage of customers is always ensured. $p$-center location problems are widely considered in location theory, especially in public sector applications (Zanjirani Farahani \& Hekmatfar, 2009).

The Rawlsian approach can be extended to a lexicographic approach, which in addition to the worst outcome maximizes the second worst (provided that the worst outcome is as large as possible), third worst (provided that the first and second worst outcomes are as large as possible) and so on (Kostreva et al., 2004). Lexicographic maximin approach is a regularization of the Rawlsian maximin approach such that it satisfies strict monotonicity and PD. Lexicographic approaches are used in Vossen and Ball (2006), Luss (2010), Luss (2008), Nace and Orlin (2007), Nace, Doan, Klopfenstein, and Bashllari (2008), Luss (2012a), Salles and Barria (2008), Wang et al. (2007), Wang et al. (2008), Lee and Cho (2007), Lee et al. (2004), Tomaszewski (2005), Ogryczak et al. (2005), Hooker (2010), Bonald et al. (2006) and Medernach and Sanlaville (2012). Lexicographic approaches are very inequality averse and considered by some studies as the "most equitable" solution.

\subsubsection{Inequality index based approaches}

In many studies equitability concerns are incorporated into the model through the use of inequality indices $I(y): \mathbb{R}^{m} \rightarrow \mathbb{R}$, which assign a scalar value to any given distribution showing the degree of inequality. Many inequality measures are studied in the economics literature (see Sen, 1973). Some of them are also used in the operational research literature when dealing with problems that involve equity concerns alongside efficiency concerns. As inequality indices are used to assess the disparity in a distribution, they are related to several mathematical concepts of dispersion and variance. They respect the anonymity property (Chakravarty, 1999) and have a value of 0 when perfect equity occurs. They assign a scalar value to the distribution (Chakravarty, 1999) and are "complete" in the sense that every pair of distributions can be compared under these measures (Sen, 1973).

The indices are used to address equitability concerns and do not incorporate any concerns on efficiency. Hence the models that use an inequality index to handle equity concerns are either designed as multicriteria models (two of the criteria usually being efficiency and equity related, respectively) or as single objective models that maximize an efficiency metric and use the index in a constraint. For example, Ogryczak (2009) works on location problems and develops bicriteria mean/equity models as simplified approaches. These models deal with the equity concern by adapting the inequality measures to the location framework and trying to minimize them. He discusses different ways to find efficient solutions to these bicriteria models. Other bi(multi)-criteria examples include Boffey et al. (2008), Kozanidis (2009), Turkcan et al. (2011), Ramos and Oliveira (2011), Jang et al. (2006), Galvão et al. (2006), Chan et al. (2004), Blakeley et al. (2003), Wu et al. (2012), Ohsawa et al. (2008) Al-Yakoob and Sherali (2006), Stolletz and Brunner (2012), Tsai and Li (2009), Martin et al. (2013), Bertsimas et al. (2014), Lejeune and Prasad (2013), Bergey et al. (2003). There are also single objective models where equity is handled via constraints and an efficiency metric is maximized (Chang et al., 2006; Mclay \& Mayorga, 2013). For example, in Mclay and Mayorga (2013) minimum levels of allocation are set for each entity using constraints.

Using an explicit inequality measure has some advantages such as bringing transparency to the decision making process, making the equitability concept computationally tractable, and hence making it possible to optimize the system with respect to these equality measures once a suitable measure is agreed upon, or to tradeoff equity and efficiency (see e.g. Zukerman et al., 2008). On the other hand, using an inequality index to incorporate equitability concerns implies a certain approach to fairness dictated by the axioms underlying the selected index and sometimes may result in oversim- plification of the discussion on equity. Moreover, different indices are based on different concepts of equity, hence may provide different rankings for the same set of alternatives. Selecting an index in line with the DM's understanding of fairness requires some extra knowledge of the underlying theoretical properties of different indices.

Recall that the widely-accepted Pigou-Dalton principle of transfers (PD) states that any transfer from a poorer person to a richer person, other things remaining the same, should always increase the inequality index value. That is, for any inequality index $I(y): \mathbb{R}^{m} \rightarrow \mathbb{R}$ satisfying PD the following holds: $y_{j}>y_{i} \Rightarrow I(y)<I\left(y+\varepsilon \mathbf{e}_{j}-\varepsilon \mathbf{e}_{i}\right)$, for all $y \in \mathbb{R}^{m}$, where $\varepsilon>0$, where $\mathbf{e}_{i}, \mathbf{e}_{j}$ are the $i$ th and $j$ th unit vectors in $\mathbb{R}^{m}$. A weak version of this principle requires such a transfer not to decrease the inequality index value. This weak version can be considered as the minimal property to be expected from an inequality index. All the indices discussed below satisfy the weak PD. We will indicate the indices that additionally satisfy (the strong version of) PD.

We now discuss the most commonly used inequality indices. All the indices except the last one are familiar from the economics literature.

(1) The range between the minimum and maximum levels of outcomes $\left(\max _{i} y_{i}-\min _{i} y_{i}\right)$ : This is the difference between the maximum and minimum outcomes in a distribution. This index is used in Boffey et al. (2008), Kozanidis (2009), Turkcan et al. (2011), Mclay and Mayorga (2013), Stolletz and Brunner (2012), Martin et al. (2013) and Kimbrel et al. (2006).

Ramos and Oliveira (2011) minimize the function $\left(\frac{\max _{i} y_{i}-\min _{i} y_{i}}{\min _{i} y_{i}}\right) \times 100$, hence use a range function normalized by the minimum outcome. A related measure, $\left(\frac{\min _{i} y_{i}}{\max _{i} y_{i}}\right)$, is used in Chang et al. (2006), which is restricted to be larger than or equal to a predefined parameter in a constraint (the constraint is of the form: $\min _{i} y_{i} \geq \eta * \max _{i} y_{i}$, where $\eta$ is called the fairness parameter.

In this method the equity level of an allocation is assessed by considering the two extremes; hence this index fails to distinguish allocations that have same level of extremes but different levels of the other values. In that sense, this index is rather crude but is used in many applications owing to its being simple and easy to understand.

(2) (Relative) mean deviation: This is the deviation from the mean. Note that in many cases the mean of the distribution is not known beforehand and is derived endogenously in the model. It is possible to use the total absolute deviations from the mean $\left(\sum_{i \in I}\left|y_{i}-\bar{y}\right|\right.$, where $\left.\bar{y}=\frac{\sum_{i \in I} y_{i}}{m} \mid\right)$ (Ogryczak, 2009; Eiselt \& Marianov, 2008; Martin et al., 2013; Bergey et al., 2003; Bertsimas et al., 2014; López-de-los Mozos et al., 2013) or to use the positive or negative deviations only, as in Ogryczak (2009). The mean deviation does not satisfy strong PD because it is not affected by transfers between two entities which are both above the mean or both below it.

Jang et al. (2006) use the mean square deviation $\left(\sum_{i \in I}\left(y_{i}-\right.\right.$ $\bar{y})^{2}$ ). Galvão et al. (2006) use the maximum componentwise deviation from average as a measure of inequity $\left(\operatorname{Max}_{i \in I}\left|y_{i}-\bar{y}\right|\right)$.

(3) Variance $\left(\sum_{i \in I}\left(y_{i}-\bar{y}\right)^{2} / m\right)$ : Turkcan et al. (2011), and Tsai and $\mathrm{Li}$ (2009) use variance as a measure of fairness in their models. Variance satisfies PD. Equivalently, the standard deviation is also used in some studies (Chan et al., 2004; Blakeley et al., 2003).

(4) Gini coefficient: One of the widely used income inequality measure used by the economists is the Gini coefficient owing to its respecting the PD (Ray, 1998). The Gini coefficient has the following formula: $\frac{\sum_{i \in I} \sum_{j \in J}\left|y_{i}-y_{j}\right|}{2 m \sum_{i \in I} y_{i}}$, where $I$ and $J$ denote the 
entity set. Two examples are (Lejeune \& Prasad, 2013 and Wu et al., 2012), who use the Gini coefficient in location of (service) facilities and in design of more equitable congestion pricing schemes, respectively.

(5) Sum of pairwise (absolute) differences $\left(\sum_{i \in I} \sum_{j \in J}\left|y_{i}-y_{j}\right|\right)$ : Sum of absolute differences between all pairs is considered in Ohsawa et al. (2008), Al-Yakoob and Sherali (2006) and Lejeune and Prasad (2013). Like the Gini coefficient and variance, this measure satisfies the PD. A closely related measure is the sum of square deviations between all pairs which is used in Szeto and Lo (2006).

The measures discussed so far are also discussed in the economics literature especially for assessing income inequality. The first two measures (range and relative mean deviation) are relatively crude measures and hence not as popular as the others for assessing income inequality. However, they are used in OR models arguably because these indices have simpler formulations than the others and so lead to more tractable optimization problems.

Another example that minimize deviation from a point of perfect equality is due to Bozkaya et al. (2003). In a political districting problem, Bozkaya et al. (2003) define an acceptable range around the average district population. In their mathematical model, they minimize the deviations from the average for the districts with populations outside this range. The squared deviation functions are also used (Suzuki \& Drezner, 2009).

Minimizing deviation from a predefined target, which, if satisfied, leads to the most equitable allocation, is also proposed, especially in goal programming applications. Azaiez and Al Sharif (2005) consider the deviation between the sum of actual days a nurse works from the minimum required days on in a nurse scheduling problem. They minimize the total positive deviations with the aim of obtaining an equitable workload distribution. The same approach is used to obtain an equitable distribution of day-shifts and night shifts over all nurses. Jahn et al. (2005) propose a mathematical model for a route guidance system where equity over network users is ensured by using constraints that avoid lengthy detours. Specifically, the ratio of length of any user's path to the "normal length" of the shortest path for the same origin-destination pair is restricted by a parameter and only the paths leading to ratios lower than this bound are allowed. They discuss three different choices for the normal length, which are the geographic distance, free flow travel times (travel times in an uncongested network) and travel times when the network is in user equilibrium and suggest using the latter.

A specific application of minimizing deviation from a predefined target is minimizing maximum regret in an uncertain environment, where the target is the best possible output that could be obtained in a realized scenario. López-de-los Mozos et al. (2013) consider a single facility location problem on a network where the node weights (demands) change through time (let $\left[t^{-}, t^{+}\right]$be the considered time interval). The dynamic nature of demands introduces a second dimension to the equity concerns since using a static measure (minimizing an inequality measure using demand data for a fixed time $t$ ) may not avoid inequity in some other time $t$ with different demand figures. The authors discuss two robustness criteria for the mean absolute deviation problem: In the first one, they minimize the maximum mean absolute deviation value over the time period $\left(\min _{x \in N} \max _{t \in[t-, t+]} F(x, t)\right.$, where $F(x, t)$ is the mean absolute deviation function for a location candidate $x \in N$ and time $t$ ) and in the second one they minimize the maximum regret over the time period $\left(\min _{x \in N} \max _{t \in[t-, t+]}\left[F(x, t)-F^{*}(t)\right.\right.$, where $F^{*}(t)=\min _{x \in N} F(x, t)$, the best value that could be reached at time $t$ )

As discussed above, there are many different inequality indices and selecting one implies certain assumptions on the decision maker's attitude to equity. For example, in a resource allocation environment, if the range is used then the focus is on the most and least deprived parties.

\subsubsection{Inequity-averse aggregation function based approaches}

One natural way to achieve an equity-efficiency trade-off without specifying an inequity index is to use an aggregation function of the distribution vector in the model that would encourage equitable distributions. An example would be a symmetric function under which a convex combination of two distributions which have the same functional value would achieve a higher value than these distributions (e.g. if the function is symmetric $(40,50)$ has a higher value than $(30,60)$ or $(60,30)$ ). Such a function is inequity averse in the sense that the averaging operation improves the distribution. By maximizing such aggregation (value) functions, we can avoid distributions that give some entities too much while depriving some others.

We call these approaches aggregation function based approaches. Unlike an inequality index which only focuses on the inequity in a distribution, an inequity-averse aggregation function reflects concerns for both equity and efficiency. There are several approaches to how the equity should be captured. There are studies that use value functions which are Schur-concave, (symmetric) quasi-concave or concave with the aim of obtaining equitable solutions. Note that when allocating a bad, a Schur-convex, quasi-convex or convex aggregation (cost) function is minimized.

In these approaches, one uses an aggregation function $U: \mathbb{R}^{m} \rightarrow$ $\mathbb{R}$, and modifies the original problem as follows: $\max \{U(y): y \in Y\}$ where $Y \in \mathbb{R}^{m}$ is the feasible outcome space. For a specified function form to be inequity-averse, it has to satisfy some properties. First of all, such a function should be symmetric to respect anonymity and should reflect concerns in terms of inequity-aversion and the equityefficiency trade-off. We call the set of symmetric functions that satisfy the strict Pigou-Dalton principle of transfers and strict monotonicity equitable aggregation functions.

Definition 1. An equitable aggregation function is a function $U: \mathbb{R}^{m} \rightarrow$ $\mathbb{R}$ for which the following hold:

$y^{1}<y^{2}$ then $U\left(y^{1}\right)<U\left(y^{2}\right)$, for all $y^{1}, y^{2} \in Y$, i.e. $U$ is strictly increasing with respect to every coordinate.

$U(y)=U\left(\Pi^{l}(y)\right)$, where $\Pi^{l}(y)$ is an arbitrary permutation of the $y$ vector, i.e. $U$ is symmetric.

$y_{j}>y_{i} \Rightarrow U(y)<U\left(y-\varepsilon \mathbf{e}_{j}+\varepsilon \mathbf{e}_{i}\right)$, for all $y \in \mathbb{R}^{m}$, where $0<\varepsilon<$ $y_{j}-y_{i}$, where $\mathbf{e}_{i}, \mathbf{e}_{j}$ are the $i^{\text {th }}$ and $j^{\text {th }}$ unit vectors in $\mathbb{R}^{m}$, i.e. U satisfies $P D$.

All equitable aggregation functions are strictly Schur-concave (Kostreva et al., 2004). Similarly, in a minimization environment, for example in cost distribution, equitable aggregations are Schurconvex functions. We now give the definition of Schur-concave functions. Let us first give the definition of a bistochastic matrix.

Definition 2. A bistochastic (doubly stochastic) matrix ( $Q$ ) is a square matrix which has all nonnegative entries and each row and column of the matrix adds up to 1.

Permutation matrices, which reorder the elements of a vector, are special cases of bistochastic matrices.

The well-known Birkhoff-von Neumann theorem (Birkhoff, 1946) states that the set of doubly stochastic matrices of order $m$ is the convex hull of the set of permutation matrices of the same order. Moreover, the vertices of this polytope are the permutation matrices. That is, a bistochastic matrix of order $m$ is a convex combination of the set of permutation matrices of the same order.

Definition 3. A functionf is strictly Schur-concave (Schur-convex) if and only if for all bistochastic matrices $Q$ that are not permutation matrices, $f(Q x)>f(x)(f(Q x)<f(x))$.

Schur-concave functions are symmetric by definition. Schurconcavity relates to more familiar concavity concepts in the following 
way: All symmetric (strictly) quasi-concave and symmetric (strictly) concave functions are (strictly) Schur-concave.

Maximizing (minimizing) a specific (strictly) Schur-concave (convex) function that aggregates the outcomes is discussed in a number of papers in the literature. Ball et al. (2009) investigate a class of models for assigning flights to slots in ground delay problems and discuss the use of Schur-convex aggregation functions as a way of obtaining equitable solutions within this setting.

Marín et al. (2010) use "ordered median functions" as objective functions of discrete location problems. Ordered median functions are weighted total cost functions, in which the weights are rankdependent. As the weights are rank dependent, these functions are symmetric and if the weights are chosen appropriately, ordered median functions can be inequity-averse in the sense that they are strictly concave. They show that both the range and sum of pairwise differences functions can be modeled using this approach, hence are particular cases of their model. Similarly, López-de-los Mozos et al. (2008) consider the ordered absolute deviation model, whose objective function is the ordered weighted average of the absolute deviations from facilities to the median value (For a candidate location at $x$, denote its distance to a demand node $i$ as $d_{i}(x)$. The cost function for demand node $i$ with a certain fraction of demand $w_{i}$ is $y_{i}(x)=w_{i}\left|d_{i}(x)-M(x)\right|$, where $M(x)=\sum_{i \in I} w_{i} d_{i}(x)$. The objective function used is $\sum_{i \in I} \lambda_{i} y_{(i)}(x)$, where $y_{(1)}(x) \leq y_{(2)}(x) \leq \ldots \leq y_{(m)}(x)$ and $\lambda_{i} \geq 0 \forall i$. Some specific cases of this formulation are mean $a b$ solute deviation for $\lambda_{i}=0 \forall i$ and maximum absolute deviation $\lambda_{i}=0$ $\forall i \neq m$ and $\lambda_{m}=1$ ). They discuss the models for cyclic, tree and path networks.

Martin et al. (2013) minimize a convex function of the form $\sum_{i \in I}$ $y_{i}^{2}$ to ensure that violations of soft constraints in nurses' rosters are equitably distributed across nurses in a scheduling problem. Similarly, Kunqi et al. (2007) maximize an additive concave utility function of the form $U=\sum_{i \in I}-W_{i}^{h} / h$ for $h \geq 1$, where $W_{i}$ deotes the waiting time user $i$ in a wireless network and $h$ is a parameter.

In communication engineering, one of the commonly used fairness concepts is proportional fairness, which can be obtained by maximizing $\sum_{i \in I} \log \left(y_{i}\right)$. An allocation $y$ is proportionally fair if for any other feasible allocation $y^{\prime}$, the total proportional change $\left(\sum_{i \in I}\left(y_{i}^{\prime}-y_{i}\right) / y_{i}\right)$ is zero or negative when all outcomes are nonnegative. The proportional fairness concept can be advocated from a game theoretic point of view as a proportionally fair allocation is also the Nash bargaining solution, satisfying certain axioms of fairness (Bertsimas et al., 2011; Crowcroft \& Oechslin, 1998; Kelly, Maulloo, \& Tan, 1998; Morell et al., 2008; Bonald et al., 2006; Kelly, Massoulié, \& Walton, 2009; Walton, 2011); see also Köppen (2013), Köppen, Yoshida, Ohnishi, and Tsuru (2012) for a discussion of proportional fairness within a relational framework and a symmetric version of this concept, -rank- ordered proportional fairness). Proportional fairness is a specific case of a more general fairness scheme called $\alpha$-fairness, which maximize the following parametric class of utility functions for $\alpha \geq 0$ (Bertsimas et al., 2012) (see also Verloop, Ayesta, and Borst (2010) for a discussion of $\alpha$-fairness in multi-class queuing systems):

$U_{\alpha}(y)=\left\{\begin{array}{l}\sum_{i=1}^{m} \frac{y_{i}^{1-\alpha}}{1-\alpha} \text { for } \alpha \geq 0, \alpha \neq 1 \\ \sum_{i=1}^{m} \log \left(y_{i}\right) \text { for } \alpha=1\end{array}\right.$

Lexicographic maximin approach, which is a regularization of the Rawlsian maximin approach such that it satisfies strict monotonicity and PD, is another example (see Medernach \& Sanlaville, 2012 for an interesting extension of this approach to resource allocation settings where the demand of the users is uncertain, modeled by scenarios. The authors propose a multicriteria approach where each scenario is treated as one criterion, i.e. the criterion is the performance of the allocation policies under one precise scenario. They try to find solutions that are Pareto optimal fair, i.e. that is maximal for the lexicographic order).

There are also approaches that use a Schur-concave function and hence respect the weak version of the PD only while failing to satisfy the strong version. For example, Hooker and Williams (2012) consider allocation of utilities to individuals (or classes of individuals) and propose a weakly Schur-concave aggregation function to be maximized. The function is based on the idea of combining objectives of equity - they use a Rawlsian approach- and efficiency. The authors provide a mixed integer linear programming formulation of the allocation problem and apply the formulation to a healthcare planning example.

A difficulty with equitable aggregation functions is that the decision maker or modeler has to select a specific aggregation function. In most settings there may not be a natural choice of equitable aggregation. A set of approaches based on the concept of a unanimity order have been developed to address this issue. Given a set $F$ of functions $f \in F$, the unanimity order with respect to $F$ is the binary relation $<^{*}$ over outcome vectors and defined as follows: for any two allocation vectors $y^{1}$ and $y^{2} \in Y, y^{1}<* y^{2} \Longleftrightarrow f(x)<f(y)$ for all $f \in F$.

Note that unanimity order is a quasiorder. The approaches discussed so far in this section maximize a particular concave, quasiconcave and Schur-concave function in their models. We note that rather than using specific functions, if we consider the unanimity order for the set of all concave, quasi-concave or Schur-concave functions, there is no difference between the resulting order. This important result is summarized in the following theorem.

Theorem 4. For two allocation vectors $y^{1}$ and $y^{2}$, the following cases are equivalent:

1. $U\left(y^{1}\right) \leq U\left(y^{2}\right)$ for all $U: U$ is increasing and Schur-concave Shorrocks (1983). (Note that Shorrocks (1983) uses a strict version of the PD; hence strictly Schur-concave functions)

2. $U\left(y^{1}\right) \leq U\left(y^{2}\right)$ for all $U: U$ is symmetric, increasing and quasiconcave (Rothschild \& Stiglitz, 1973)

3. $U\left(y^{1}\right) \leq U\left(y^{2}\right)$ for all $U: U$ is symmetric, increasing and concave (Rothschild \& Stiglitz, 1973)

4. $U\left(y^{1}\right) \leq U\left(y^{2}\right)$ for all $U$ : $U$ is additive, increasing and concave. That is, $U(g)=\sum_{i \in I} u\left(y_{i}\right)$ where $u$ is increasing and concave (Shorrocks, 1983; Rothschild \& Stiglitz, 1973)

Parts of Theorem 4 for the special case where $\sum_{i \in I} y_{i}^{1}=\sum_{i \in I} y_{i}^{2}$ are proven by Atkinson (1970) and Dasgupta, Sen, and Starrett (1973) based on the results by Hardy, Littlewood, and Polya (1934) on majorization (see also Marshall, Olkin, \& Arnold, 2009). The results for the more general case $\left(\sum_{i \in I} y_{i}^{1} \neq \sum_{i \in I} y_{i}^{2}\right)$ can be found in Rothschild and Stiglitz (1973) and Shorrocks (1983). This theorem states that the unanimity ordering of a given set of alternatives under the set of all Schur-concave functions is equivalent to the unanimity ordering under the set of all quasiconcave, concave functions or additive functions of concave functions.

Some studies that design allocation systems over multiple periods optimize an aggregation function at each period. They make sure that the aggregation is inequity-averse by updating its parameters. Such an approach is used in Jeong et al. (2005) in a data traffic scheduling algorithm where the time is divided into multiple periods and at each period a weighted sum of transmit data rates of the users of the system is maximized. The weight for each customer is updated at the beginning of each period to control the level of fairness, i.e. a larger weight is assigned to a user whose previous receiving data rate was low. As an example, they suggest using $w_{i}=A+B e^{-\operatorname{Ravg}(i)}$, where $A$ and $B$ are constants and $\operatorname{Ravg}(i)$ is the average data rate of user $i$ up to the scheduling period considered. Alsheddy and Tsang (2011) use the same idea in assigning jobs to staff over multiple periods. They try to find an assignment that is in line with employees' individual submitted plans by maximizing the number of such plans satisfied in 
the solution. At each day $d$ the objective function used is of the following form: Max $\frac{\sum_{i \in I} x_{i} w_{i}}{\sum_{i \in I} w_{i}}$, where $x_{i}$ is 1 if the workplan of employee $i$ is satisfied and $w_{i}$ is the weight given to this workplan, which is updated through time in a way that gives more decision power to the employees who experienced a low number of satisfied plans so far (on a day $d, w_{i}=\left(d-s a t_{i}\right) / d$, where $s a t_{i}$ is the number of satisfied plans on previous days for employee $i$ ).

\subsubsection{A multicriteria perspective: equitable efficiency and \\ Schur-concavity}

The above approaches use particular functions in order to capture equity concerns. The specific functional forms used are context dependent and different forms are adopted in different studies. Two common properties of these functions are that they are increasing or nondecreasing (in a maximization problem) and inequity-averse in the sense that they satisfy PD, though sometimes in a weak way as in Hooker and Williams (2012). Considering the aggregation function approach from a multicriteria perspective, one can relate such functions to the DM's preferences and specify a set of properties that an equity-averse DM's preference model should satisfy. Kostreva and Ogryczak (1999) and Kostreva et al. (2004) take this point of view and introduce the concept of equitable efficiency. Given two distributions, the "more equitable" one is distinguished based on a set of axioms defined on the DM's preference model. They call a social welfare function which is in line with this specific set of axioms an equitable aggregation function and a solution which maximizes an equitable aggregation function equitably efficient. This multicriteria decision making perspective is based on defining each element of the outcome vector as a separate criterion to be maximized as explained below. This discussion is based on the theory introduced in Kostreva and Ogryczak (1999).

Consider the following problem: $\max \{f(x): x \in Q\}$ where $X \in \mathbb{R}^{n}$ is the decision space, $Y \in \mathbb{R}^{m}$ is the outcome space and $f(x)$ is a vector function that maps $X$ to $Y$ and $Q$ is the feasible set. A typical outcome vector is $y^{k}=\left(y_{1}^{k}, y_{2}^{k}, \ldots, y_{m}^{k}\right)$, where $y_{i}^{k}$ is the outcome value corresponding to entity $i \in I(i=1,2, \ldots, m)$ and $k$ is the index of the alternative.

We denote the weak preference relation of the DM as $\preceq$ (the corresponding strict and indifference relations are denoted by $\prec$ and $\sim$, respectively). Assume that the DM has a preference model in which the preference relation satisfies the following axioms (Kostreva et al., 2004):

1. Reflexivity $(R): y \preceq y$ for all $y \in Y$.

2. Transitivity (T): $\left(y^{1} \preceq y^{2}\right.$ and $\left.y^{2} \preceq y^{3}\right) \Rightarrow y^{1} \preceq y^{3}$, for all $y^{1}, y^{2}$, $y^{3} \in Y$.

3. Strict monotonicity (SM): $y^{1}<y^{2}$ then $y^{1} \prec y^{2}$, for all $y^{1}, y^{2} \in Y$.

4. Anonymity $(A)$ : $(y) \sim \Pi^{l}(y)$ for all $l=1, \ldots, m$ !, for all $y \in \mathbb{R}^{m}$, where $\Pi^{l}(y)$ stands for an arbitrary permutation of the $y$ vector.

5. Pigou-Dalton principle of transfers (PD): $y_{j}>y_{i} \Rightarrow y \prec y-\varepsilon \mathbf{e}_{j}+\varepsilon \mathbf{e}_{i}$, for all $y \in \mathbb{R}^{m}$, where $0<\varepsilon<y_{j}-y_{i}$, where $\mathbf{e}_{i}, \mathbf{e}_{j}$ are the $i$ th and $j$ th unit vectors in $\mathbb{R}^{m}$.

The anonymity axiom states that the corresponding preference relation should treat all the permutations of a vector as indifferent. That is, the identities of the entities are irrelevant. This is in contrast to what we have called balance problems. The preference for equity is stated by the PD axiom. The preference relations that satisfy $\mathrm{R}$, $\mathrm{T}, \mathrm{SM}, \mathrm{A}$ and $\mathrm{PD}$ are called equitable rational preference relations. Using equitable rational preference relations, the relations of equitable dominance, equitable indifference and equitable weak dominance can be defined as follows:

Definition 5. For any two outcome vectors $y^{1}$ and $y^{2}$,

$y^{1} \prec_{e}\left(/ \preceq_{e} / \sim_{e}\right) y^{2}\left(y^{2}\right.$ equitably dominates/ equitably weakly dominates/is equitably indifferent to $\left.y^{1}\right)$ iff $y^{1} \prec(/ \preceq / \sim) y^{2}$ for all equitable rational preference relations $\preceq$.
Note that "rational dominance", i.e. the normal dominance concept, which is the intersection relation of all preference relations satisfying $\mathrm{R}, \mathrm{T}$ and $\mathrm{SM}$, implies equitable dominance, but not vice versa.

Equitable dominance is also called generalized Lorenz dominance (see Shorrocks, 1983). Generalized Lorenz dominance is an extension of the well-known Lorenz dominance concept used in the economics literature to the cases where the means of the distributions are not necessarily equal. An alternative is equitably efficient if there is no alternative that equitably dominates it. Note that the set of equitably efficient solutions is a subset of the Pareto efficient set.

We have already defined (see Definition 1) equitable aggregation functions. It so happens that the equitable aggregations, i.e. Schurconcave functions are the functions that respect axioms $1-5$. That is, if an equitable rational preference relation is representable by a utility function, the function has to be increasing strictly Schur-concave in a maximization problem (Kostreva et al., 2004). The equitably efficient set is the set of alternatives each of which maximizes at least one increasing strictly Schur-concave function.

There are two possible equity modeling approaches using such aggregations: The first approach is choosing a suitable equitable aggregation function (Schur-concave function) and optimizing it in the model. Optimizing a predefined aggregation function will return one of the (possibly many) equitably efficient solutions. The aggregation function based approaches discussed previously, which optimize a strictly Schur-concave (Schur-convex) function are in this category.

The second approach is finding the set of equitably efficient solutions without specifying the aggregation function further. This way one would obtain a set of alternatives that is guaranteed to include the DM's most preferred alternative as long as her utility function is (strictly) Schur-concave. This approach is discussed in Kostreva and Ogryczak (1999) and Kostreva et al. (2004) for multiple criteria linear problems and nonlinear problems, respectively. Baatar and Wiecek (2006) define the equitable preference structure using a cone-based approach and propose a two step method including two single objective nonlinear programs in order to find equitably efficient solutions.

As an application example, Ogryczak et al. (2008) consider equitable optimisation in bandwidth allocation. For practical purposes, they consider a restricted set of criteria and find equitable solutions for the restricted model using the reference point approach. A similar approach is taken in Ogryczak (2007).

Mut and Wiecek (2011) generalize the concept of equitability. They define two different relations which are more general than $\preceq_{e}$ and investigate the axioms that these new relations satisfy. They derive the conditions under which the new preferences satisfy the original and modified axioms of equitable preference.

In most of the above approaches the whole set of nondominated points or a subset of it is found; hence the algorithms return multiple alternatives without using an interactive setting. The studies we encountered that consider interactive approaches are Kostreva et al. (2004) and Ogryczak et al. (2008), which use a reference point approach and Karsu, Morton, and Argyris (2012), which use the convex cones approach to incorporate DM's preference information to guide the selection or ranking process.

The classical multicriteria decision making problem settings include criteria that do not have the same range, hence it is not appropriate to use equitable aggregation over the original criteria values. However, in the reference point method, the outcome vectors are converted to achievement vectors using scalarizing functions. The scalarizing function transforms the outcomes into a uniform scale, which makes it possible to apply an equitable aggregation on the transformed achievement scores. Kostreva et al. (2004) make this observation and discuss the use of equitable aggregations for the reference point method. Using the same idea, Dugardin et al. (2010) use the equitable dominance concept in a well-known multi-criteria solution approach (NSGA2) to discard the alternatives which are competitive in only one criterion. The authors introduce a function which 
scales different components of the objective vector. This is an application where the equity concept is used in order to choose "good" alternatives in a multi-criteria problem that does not have the impartiality property. These applications show the two way link between the Pareto efficiency and the equitable efficiency concept. In addition to generating equitably efficient solutions using the classical MCDM solution methods designed to generate Pareto efficient solutions, one can also use the equitable efficiency concept to come up with Pareto efficient solutions once the outcome vectors are modified using appropriate scalarizations.

\subsection{Handling balance}

Most of the approaches handle balance concerns by using an imbalance indicator, which measures deviation from a predefined level, which is chosen e.g. based on claims, needs or preferences. This approach is similar to an inequality index based approach to equitability, however an imbalance indicator does not necessarily achieve its minimum at a distribution where each entity receives the same amount.

Examples of applications handling the balance concept using this approach are as follows. In a heterogeneous server system model, Armony and Ward (2010) consider equity over servers with different service rates. They formulate the problem as a Markov decision process and solve a related LP model, in which the customer waiting time is minimized along with a fairness constraint on the workload division over servers with different skill levels. Specifically, they use a constraint set that controls the fraction of the idle time that the server groups with different paces have. These fractions are ensured to have pre-determined values, which are set by the decision maker. Similarly, Ward and Armony (2013) set idleness ratios of server pools in a queueing system in constraints.

Cook and Zhu (2005) allocate a fixed cost among the existing Decision Making Units (DMU). In order to treat the DMUs in an equitable way, the authors ensure that the efficiencies of the DMUs remain unchanged after the allocation. Smith et al. (2009) and Smith et al. (2013) incorporate balance concerns over users of a public service provision system by minimizing weighted negative and positive deviations from a standard service level specified by the DM.

Avi-Itzhak, Levy, and Raz (2007) and Avi-Itzhak et al. (2008) discuss a resource-allocation based fairness measure, which assumes that every customer in a queuing system is entitled to an equal share of the resource (server). Hence the discrimination of a customer $i$ (with a service requirement $s_{i}$ ) who arrives in apoch $a_{i}$ and departs at epoch $d_{i}$ is as follows: $s_{i}-\int_{a_{i}}^{d_{i}}(1 / m(t)) d t$, where $m(t)$ is the number of customers in the system. If the unfairness of a specific scenario is of concern, it can be calculated by taking summary statistics over all discriminations in that scenario. If the system unfairness is of concern, then the proposed unfairness measures are the variance or expected absolute value of discrimination (as the expected value is zero).

In ground delay programs, the ration-by-schedule (RBS) rule is used as a reference. This rule assigns the landing slots to unassigned flights on a First Scheduled First Served (FSFS) basis based on the arrival times submitted at the beginning of the daily operations. The studies that use the deviation from the FSFS solution as a measure of inequity (imbalance) in arrival slot allocations are Ball et al. (2010), Balakrishnan and Chandran (2010), Barnhart et al. (2012) and Glover and Ball (2013). In a queuing system Avi-Itzhak et al. (2008) review two such seniority based fairness indicators, where FCFS rule is taken as the most equitable rule and inequity (imbalance) is quantified using measures of deviation from this schedule. In the first one the deviation is quantified by the number of slips (occurs when the customer of concern overtakes another customer who arrived earlier) and skips (occurs when the customer of concern is overtaken by another customer). In the second measure the following quantity is used: $c \sum_{i \in I} a_{i} \Delta_{i}+\alpha$, where $a_{i}$ is the arrival epoch of customer $i, \Delta_{i}$ is the order displacement of customer $i$, i.e., the number of positions customer $i$ is pushed ahead or backward in the schedule compared to the his position at the FCFS order and $c>0$ and $\alpha$ are arbitrary constants. They report that under steady state this quantity is equivalent to the variance of the waiting time, up to a constant multiplier. Another measure takes into account both order violation (in the form of skips) and size violation events, which occur when upon arrival, a customer finds that another customer whose residual service is greater than or equal to the service requirement of himself/ herself, departs earlier or concurrently. The total number of such order and size violations is used as a measure of unfairness (Sandmann, 2013). In a restaurant revenue management problem (Bertsimas \& Shioda, 2003) ensure equity across customers of the same size by using constraints that seat them on a FCFS basis.

Karsu and Morton (2014) propose a two dimensional framework to trade balance off against efficiency in resource allocation problems motivated by problems in R\&D project selection. They use imbalance indices which measure the deviation of an allocation from an ideally balanced allocation the DM provides.

The deviation (cost) function, i.e. the imbalance indicator, can be the total absolute deviation (Glover \& Ball, 2013) or the sum of negative or positive deviations. There are also studies that minimize the maximum componentwise deviation (Ball et al., 2010; Vitoriano et al., 2011) or use a constraint which ensures that maximum componentwise deviation is below a pre-defined level (Smith et al., 2011; Balakrishnan \& Chandran, 2010). In some models designed to improve an existing system (e.g. the current transportation network) any negative deviation from the status quo is forbidden by constraints as in Lo and Szeto (2009). They propose a transportation network improvement model, which ensures that no origin-destination pair gets worse than the status quo in terms of consumer surplus, i.e. the difference between what travelers would be willing to pay for travel and what they actually pay. There are also studies that use a weighted total deviation from the weighted mean such as Sherali et al. (2003, 2006, 2011) $\left(\sum_{i \in I} w_{i}\left|y_{i}-\sum_{i \in I} w_{i} y_{i}\right|\right.$, where $\left.\sum_{i \in I} w_{i}=1\right)$.

The above studies focus on keeping the total deviation from a predefined level at minimum, which may result in some componentwise deviations to be significantly larger than others. Similar to equitable aggregation functions, convex functions are optimized in some models to handle balance concerns. Such convex functions encourage fairness in the distribution of deviation (cost) and hence avoid some entities deviate significantly for the sake of minimizing total deviation. In that sense, convex functions can be considered as special types of imbalance indicators, which measure deviation using a convex function. Exponential (cost) functions and squared deviation functions are examples of such convex functions (Mukherjee \& Hansen, 2007; Suzuki \& Drezner, 2009). Mukherjee and Hansen (2007) propose a dynamic stochastic integer programming model for the GDP that allows one to revisit the assignment in case of a change in airport operating conditions. They use a convex ground delay cost function in their objective in order to ensure a uniform spread of ground delay across different flight categories. Kotnyek and Richetta (2006) consider the stochastic GDP and ensure that the FSFS holds by using convex ground-hold cost functions. Lulli and Odoni (2007) use the same idea in an Air Traffic Flow Management model, where an equitable distribution of delay is achieved by using objective function cost coefficients that are a convex function of the tardiness of a flight. Similarly, Barnhart et al. (2012) use an exponential delay penalty function. For each flight a worst-case FSFS delay is calculated and each interval delay beyond this worst-case FSFS delay is penalized by an exponentially increasing amount. Similarly, Bollapragada and Garbiras (2004) minimize a piecewise linear penalty function of deviations from goals. In an access control policy design problem, Zhang and Shen (2010) incorporate spatial equity into the model by using the weighted square sum of the average delay over different entry points. Huang et al. (2012) use convex disutility functions of unsatisfied demand percentages of 
each node in a relief routing model. Hence, the whole demand of each node is not necessarily supplied so as to save supply for other nodes.

It is also possible to use a scaling approach and define the outcome distribution as the per capita allocation, i.e. $y_{i} / n_{i}$ where $n_{i}$ is an attribute value, such as a measure showing the size or need of an entity. For example in disaster relief models, the proportion of demand satisfied in different demand nodes is used as a measure of service (Davis et al., 2013; Vitoriano et al., 2011; Tzeng et al., 2007). This scaling approach allows one to assume anonymity over the scalarized outcome distribution and hence handle the balance concerns in an equitability environment. Examples that use this scaling approach are used in different settings including public policy (Johnson et al., 2010; Johnson, 2003), drug allocation to clinics (Swaminathan, 2003; Swaminathan et al., 2004), water resources allocation (Wang et al., 2007; Wang et al., 2008), sequential resource allocation (Geng et al., 2014), transportation network design (Szeto \& Lo, 2006) and scheduling (Higgins \& Postma, 2004)

\section{Conclusion}

Although most (of the early) attempts in operational research focused on efficiency concerns, there is a vast amount of applications where equity is an additional concern. The need for equity is appreciated by the OR practitioners and academicians as can be observed by the recent increase in the number of OR papers, which re-consider some of the well-known problems such as knapsack, assignment and location problems with an effort to incorporate equity concerns. The applications that require explicit consideration of equity appear in a broad range of situations both in the public and private sector.

In this paper, we provide a review of the approaches that are used to handle equity concerns by optimizing mathematical models. We focus on the studies that consider equity alongside other, mostly efficiency-related, concerns. We first discuss two equity related concepts: equitability, and balance. We discuss the differences between these two concepts along with their applications. Most of the approaches in our review can be classified as either being concerned with equitability, i.e. assuming anonymity or with balance, i.e. distinguishing entities with respect to an attribute indicating for example need, claim or preference. Handling equity by promoting diversity is an indirect approach which is discussed only in a few papers and it is possible to define such diversity concerns as a special case of balance or equitability concerns.

We provide a detailed discussion of the solution approaches designed to incorporate equitability and balance concerns. We categorise the solution approaches to problems involving equitability concerns into three categories. The first and the crudest approach is the Rawlsian (maxmin) approach, which compares alternative distributions based on the amount the worst-off entity receives. In the second approach, an inequality measure is used either in a constraint or as a criterion so as to quantify equity. When the inequality index is used in a constraint in the model, inequity is kept below a certain threshold by this constraint. The inequality measure can also be defined as a separate criterion alongside other efficiency related criteria, resulting in a multi-criteria model. Bicriteria equity/efficiency models defined this way are easy to solve. Inequality indices are useful as summary measures but should be used with caution as they may lead to oversimplification of the equity concept. Understanding the strengths and weaknesses of each index and choosing the most appropriate one requires some knowledge of the underlying theory of inequality measurement.

The last approach to equitability is based on using inequity-averse aggregation functions of the outcome distributions. Some studies using this approach maximize specific inequity-averse functions in their models. Multicriteria decision making concepts provide us with a means to relate a set of inequity-averse functions with a set of axioms on the underlying preference relation of a DM. Two equity-related axioms are additionally assumed for a rational DM's preference relation: anonymity and the Pigou-Dalton principle of transfers. The set of functions that represent such preference relations are called equitable aggregations and all such functions are Schur-concave in a maximization problem. These aggregations can be used as scalarizing functions to obtain the set of nondominated (equitably efficient) solutions or as the single objective function to be optimized to obtain a specific nondominated solution.

Balance concerns are handled in two main ways. The first one is based on using imbalance indicators, which measure the deviation from a reference solution which is considered as balanced. These indicators can be functions of various forms including convex deviation functions, which distribute deviation in an equitable way across the entities. The second way to handle balance concerns is to convert balance problems into equitability problems by normalising allocations, hence making it possible to use any of the equitability-handling approaches.

Among the approaches used to handle equitability concerns, finding the set of equitably efficient solutions can be used as a "gold standard" for other approaches owing to its reasonably weak assumptions on the underlying preference relation (the DM can have any type of Schur-concave function). This multicriteria approach is more attractive than an inequality index based approach as specifying an inequality index may be difficult for the DM. On the other hand, the approaches that find the set of equitably efficient solutions are computationally complex. One way to choose from these two extremes would be relying on the equitable aggregation concept when the underlying optimisation problem at hand is relatively simple and easy to solve; and using an inequality index when the problem is less tractable.

We see great potential for further research in improving the decision support process in multicriteria problems where equity is a concern. Further research on guiding the DM through the set of candidate alternatives (e.g. the nondominated alternatives) could be usefully performed. This applies to multicriteria models in both inequality (or imbalance) index based and aggregation function based approaches. Selecting the "best" alternative requires information on the tradeoff between the criteria unless there is a single alternative which is better than the others in terms of all criteria, which is unlikely. Hence, in most of the multicriteria mathematical modeling approaches which address equity concerns using inequality or imbalance indices, a single alternative is obtained by maximizing a weighted sum of the criteria with predetermined weights. A more robust approach would be presenting the DM with a subset of solutions or using an interactive procedure rather than predefined weights. Which approach is more appropriate depends on the problem context. In some cases, presenting the DM with a subset of "good" solutions for further evaluations may be required from the analyst whereas in some others decision support may be required until the decision maker makes the final selection. Similarly, in equitable aggregation based multicriteria models, even if some or all the equitably efficient solutions are found and presented to the DM, it may be difficult for him to choose from this set. Appropriate decision support would be required if the decision maker wants to obtain a single solution. This renders interactive approaches relevant and necessary in such settings.

Most of the problems in OR can be categorized into one of three classes based on what is required from the decision support. These are finding the best solution (or a subset of good solutions), ranking and sorting (Roy, 1971; Figueira, Greco, \& Ehrgott, 2005). All the papers in our review of the operational research literature consider the first type although there may be ranking or sorting problems in which equity should be considered. An example of a ranking problem involving equity concerns arises naturally in intercountry comparisons based on income inequality and social welfare. This is one of the classical topics in the theory of equity as it has been discussed in economics. MCDM optimization tools can be relatively easily adapted for ranking and sorting problems that involve equity concerns: See Sen (1973) for 
a discussion and Karsu et al. (2012) for an interactive ranking algorithm that is based on the equitable efficiency concept. An interesting application would be finding ways to sort different countries in terms of social welfare, or to sort different policy decisions in terms of the resulting social welfare.

In many cases addressing fairness concerns results in a decrease in efficiency. A relevant question is how much one sacrifices from efficiency when a "fair" solution is adopted. Observing the tradeoff between efficiency and equity would make the DMs more comfortable when making decisions and communicating the decisions to the stakeholders. For example, if the efficiency loss is negligible, the DM would find it easier to support a solution that ensures fairness. On the other hand if the efficiency loss is significant, a compromise solution can be selected. There are studies in the literature that analyze the price of fairness, i.e. the efficiency difference between the following two cases: selecting a very aggressive inequality averse approach and not using an inequality averse approach (Bertsimas et al., 2011; Bertsimas et al., 2012). This concept can be generalized to see the extend to which selecting the "wrong" inequality approach affects the solutions. Analyzing robustness of solutions with respect to different inequity-averse approaches awaits further attention. There are some initial attempts to explore the similarities of different inequality measures used in the location context (see e.g. Mulligan, 1991; Lopez-de-los mozos \& Mesa, 2003 and other references therein) but there is still more research to be done. As pointed out in Lopez-delos mozos and Mesa (2003) an axiomatic introduction of the equality (and imbalance) measures could throw some light on the question of how similar different measures are. Even when an inequality or imbalance index is chosen and used in a constraint, which controls its value by a threshold, sensitivity analysis can be performed to see the effect of the threshold value on the optimal solution. Such an analysis would help us to suggest more robust solutions but was not discussed in most of the studies (see Batta et al. (2014) for an analysis in the context of a p-median problem on a network, where the authors try to find how bad a locational choice can be provided that the decision makes use dispersion, population and equity criteria).

To sum up, we believe that being a practically relevant and theoretically challenging concept, equity can stimulate a number of research questions for operational researchers especially in the areas of decision support, different problem types, and robustness.

\section{Acknowledgments}

We are thankful to the anonymous referees for their constructive comments which led to substantial improvement of the paper.

\section{References}

Al-Yakoob, S. M., \& Sherali, H. D. (2006). Mathematical programming models and algorithms for a class-faculty assignment problem. European Journal of Operational Research, 173(2), 488-507.

Alsheddy, A., \& Tsang, E. P. K. (2011). Empowerment scheduling for a field workforce. Journal of Scheduling, 14(6), 639-654.

Angel, E., Bampis, E., \& Pascual, F. (2008). How good are SPT schedules for fair optimality criteria. Annals of Operations Research, 159, 53-64.

Aringhieri, R. (2009). Composing medical crews with equity and efficiency. Central European Journal of Operations Research, 17(3), 343-357.

Armony, M., \& Ward, A. R. (2010). Fair dynamic routing in large-scale heterogeneousserver systems. Operations Research, 58(3), 624-637.

Atar, R., Shaki, Y. Y., \& Shwartz, A. (2011). A blind policy for equalizing cumulative idleness. Queueing Systems Theory and Applications, 67(4), 275-293.

Atkinson, A. B. (1970). On the measurement of inequality. Journal of Economic Theory, 2, 244-263.

Avi-Itzhak, B., Levy, H., \& Raz, D. (2007). A resource allocation queueing fairness measure: Properties and bounds. Queueing Systems, 56(2), 65-71.

Avi-Itzhak, B., Levy, H., \& Raz, D. (2008). Quantifying fairness in queuing systems. Probability in the Engineering and Informational Sciences, 22(04), 495-517.

Azaiez, M., \& Al Sharif, S. (2005). A 0-1 goal programming model for nurse scheduling. Computers \& Operations Research, 32(3), 491-507.

Baatar, D., \& Wiecek, M. (2006). Advancing equitability in multiobjective programming. Computers \& Mathematics with Applications, 52(1-2), 225-234.
Balakrishnan, H., \& Chandran, B. G. (2010). Algorithms for scheduling runway operations under constrained position shifting. Operations Research, 58(6), $1650-1665$.

Ball, M., Dahl, G., \& Vossen, T. (2009). Matchings in connection with ground delay program planning. NETWORKS, 53(3), 293-306.

Ball, M. O., Hoffman, R., \& Mukherjee, A. (2010). Ground delay program planning under uncertainty based on the ration-by-distance principle. Transportation Science, 44(1), $1-14$.

Barnhart, C., Bertsimas, D., Caramanis, C., \& Fearing, D. (2012). Equitable and efficient coordination in traffic flow management. Transportation Science, 46(2), 262-280.

Baron, O., Berman, O., Krass, D., \& Wang, Q. (2007). The equitable location problem on the plane. European Journal of Operational Research, 183(2), 578-590.

Bashiri, M., \& Tabrizi, M. M. (2010). Supply chain design: A holistic approach. Expert Systems with Applications, 37(1), 688-693.

Batta, R., Lejeune, M., \& Prasad, S. (2014). Public facility location using dispersion, population, and equity criteria. European Journal of Operational Research, 234(3), 819-829.

Beamon, B. M., \& Balcik, B. (2008). Performance measurement in humanitarian relief chains. International Journal of Public Sector Management, 21(1), 4-25.

Bell, J. E., Griffis, S. E., Cunningham, W. A., \& Eberlan, J. A. (2011). Location optimization of strategic alert sites for homeland defense. Omega, 39(2), 151-158.

Beraldi, P., Ghiani, G., Musmanno, R., \& Vocaturo, F. (2010). Efficient neighborhood search for the probabilistic multi-vehicle pickup and delivery problem. Asia-Pacific Journal of Operational Research, 27(3), 1-6.

Bergey, P. K., Ragsdale, C. T., \& Hoskote, M. (2003). A simulated annealing genetic algorithm for the electrical power districting problem. Annals of Operations Research, $121,33-55$.

Berman, O., Drezner, Z., Tamir, A., \& Wesolowsky, G. O. (2009). Optimal location with equitable loads. Annals of Operations Research, 167(1), 307-325.

Bertsimas, D., Farias, V. F., \& Trichakis, N. (2011). The price of fairness. Operations Research, 59(1), 17-31.

Bertsimas, D., Farias, V. F., \& Trichakis, N. (2012). On the efficiency-fairness trade-off. Management Science, 58(12), 2234-2250.

Bertsimas, D., Farias, V. F., \& Trichakis, N. (2013). Fairness, Efficiency, and Flexibility in Organ Allocation for Kidney Transplantation. Operations Research, 61(1), 73-87.

Bertsimas, D., Gupta, S., \& Lulli, G. (2014). Dynamic resource allocation: A flexible and tractable modeling framework. European Journal of Operational Research, 236(1), $14-26$.

Bertsimas, D., \& Shioda, R. (2003). Restaurant revenue management. Operations Research, 51(3), 472-486.

Bertsimas, D., \& Stock Patterson, S. (1998). The air traffic flow management problem with enroute capacities. Operations Research, 46(3), 406-422.

Birkhoff, G. (1946). Tres observaciones sobre el algebra lineal (three observations on linear algebra). Universidad Nacional de Tucumán Revista Serie A, 5, 147-151.

Blakeley, F., Bozkaya, B., Cao, B., Hall, W., \& Knolmajer, J. (2003). Optimizing periodic maintenance operations for Schindler elevator corporation. Interfaces, 33(1), 67-79.

Boffey, T., Mesa, J., Ortega, F., \& Rodrigues, J. (2008). Locating a low-level waste disposal site. Computers \& Operations Research, 35(3), 701-716.

Bollapragada, S., \& Garbiras, M. (2004). Scheduling commercials on broadcast television. Operations Research, 52(3), 337-345.

Bonald, T., Massoulié, L., Proutière, A., \& Virtamo, J. (2006). A queueing analysis of max-min fairness, proportional fairness and balanced fairness. Queueing Systems, $53(1-2), 65-84$.

Bozkaya, B., Erkut, E., \& Laporte, G. (2003). A tabu search heuristic and adaptive memory procedure for political districting. European Journal of Operational Research, $144,12-26$.

Briskorn, D., \& Drexl, A. (2009). A branch-and-price algorithm for scheduling sport leagues. Journal of the Operational Research Society, 60(1), 84-93.

Butler, M., \& Williams, H. P. (2006). The allocation of shared fixed costs. European Journal of Operational Research, 170(2), 391-397.

Caballero, R., González, M., Guerrero, F. M., Molina, J., \& Paralera, C. (2007). Solving a multiobjective location routing problem with a metaheuristic based on tabu search. Application to a real case in Andalusia. European Journal of Operational Research, 177(3), 1751-1763.

Campbell, A. M., Vandenbussche, D., \& Hermann, W. (2008). Routing for relief efforts. Transportation Science, 42(2), 127-145.

Cappanera, P., \& Scutella, M. G. (2005). Balanced paths in acyclic networks: Tractable cases and related approaches. Networks, 45(2), 104-111.

Caramia, M., Giordani, S., \& Iovanella, A. (2010). On the selection of k routes in multiobjective hazmat route planning. IMA Journal of Management Mathematics, 21, 239-251.

Carotenuto, P., Giordani, S., \& Ricciardelli, S. (2007). Finding minimum and equitable risk routes for hazmat shipments. Computers \& Operations Research, 34(5), 1304-1327.

Cayirli, T., \& Veral, E. (2003). Outpatient scheduling in health care: A review of literature. Production and Operations Management, 12(4), 519-549.

Chakravarty, S. R. (1999). Measuring inequality: The axiomatic approach. In J. Silber (Ed.), Recent economic thought series: Vol. 71. Handbook of income inequality measurement (pp. 163-186). Springer Netherlands.

Chan, F. T. S., Chung, S. H., \& Wadhwa, S. (2004). A heuristic methodology for order distribution in a demand driven collaborative supply chain. International Journal of Production Research, 42(1), 1-19.

Chang, K.-N., Lee, K.-D., \& Kim, D. (2006). Optimal timeslot and channel allocation considering fairness for multicell CDMA/TDD systems. Computers E Operations Research, 33(11), 3203-3218. 
Chanta, S., Mayorga, M. E., \& McLay, L. A. (2011). Improving emergency service in rural areas: A bi-objective covering location model for EMS systems. Annals of Operations Research, 221(1), 133-159.

Cook, W. D., \& Zhu, J. (2005). Allocation of shared costs among decision making units: A DEA approach. Computers \& Operations Research, 32(8), 2171-2178.

Craveirinha, J., Girão Silva, R., \& Clímaco, J. (2008). A meta-model for multiobjective routing in MPLS networks. Central European Journal of Operations Research, 16(1), 79-105.

Crowcroft, J., \& Oechslin, P. (1998). Differentiated end-to-end internet services using a weighted proportionally fair sharing TCP. ACM Computer Communications Review, 28, 53-67.

Dasgupta, P., Sen, A., \& Starrett, D. (1973). Notes on the measurement of inequality. Journal of Economic Theory, 6, 180-187.

Davis, L. B., Samanlioglu, F., Qu, X., \& Root, S. (2013). Inventory planning and coordination in disaster relief efforts. International Journal of Production Economics, 141(2), 561-573.

Dell'Olmo, P., Gentili, M., \& Scozzari, A. (2005). On finding dissimilar Pareto-optimal paths. European Journal of Operational Research, 162(1), 70-82.

Demirci, M. C., Schaefer, A. J., Romeijn, H. E., \& Roberts, M. S. (2012). An exact method for balancing efficiency and equity in the liver allocation hierarchy. INFORMS Journal on Computing, 24(2), 260-275.

Dugardin, F., Yalaoui, F., \& Amodeo, L. (2010). New multi-objective method to solve reentrant hybrid flow shop scheduling problem. European Journal of Operational Research, 203(1), 22-31.

Duran, G., \& Wolf-Yadlin, R. (2011). A mathematical programming approach to applicant selection for a degree program based on affirmative action. Interfaces, $41(3), 278-288$

Earnshaw, S. R., Hicks, K., Richter, A., \& Honeycutt, A. (2007). A linear programming model for allocating HIV prevention funds with state agencies: A pilot study. Health Care Management Science, 10(3), 239-252.

Eiselt, H., \& Marianov, V. (2008). Employee positioning and workload allocation. Computers \& Operations Research, 35(2), 513-524.

Erdogan, G., Erkut, E., Ingolfsson, A., \& Laporte, G. (2010). Scheduling ambulance crews for maximum coverage. Journal of the Operational Research Society, 61(4), 543-550.

Ernst, A., Jiang, H., Krishnamoorthy, M., \& Sier, D. (2004). Staff scheduling and rostering: A review of applications, methods and models. European Journal of Operational Research, 153(1), 3-27.

Espejo, I., Marín, A., Puerto, J., \& Rodríguez-Chía, A. M. (2009). A comparison of formulations and solution methods for the minimum-envy location problem. Computers \& Operations Research, 36(6), 1966-1981.

Figueira, J., Greco, S., \& Ehrgott, M. (2005). Multiple criteria decision analysis: State of the art surveys. USA: Springer

Galvão, R. D., Acosta Espejo, L. G., Boffey, B., \& Yates, D. (2006). Load balancing and capacity constraints in a hierarchical location model. European Journal of Operational Research, 172(2), 631-646.

Geng, X., Huh, W. T., \& Nagarajan, M. (2014). Sequential resource allocation with constraints: Two-customer case. Operations Research Letters, 42(1), 70-75.

Giovanni, A., Lorenzo, B., \& Guglielmo, G. (2000). From ground holding to free flight: An exact approach. Transportation Science, 34(4), 394-401.

Glover, C. N., \& Ball, M. O. (2013). Stochastic optimization models for ground delay program planning with equity-efficiency tradeoffs. Transportation Research Part C: Emerging Technologies, 33, 196-202.

Hardy, G. H., Littlewood, J. E., \& Polya, G. (1934). Inequalities. Cambridge.

Heikkinen, T. (2004). Power allocation in a wireless network using scheduling and stochastic programming with penalty functions. Optimization and Engineering, 5(4), 431-459.

Higgins, A., \& Postma, S. (2004). Australian sugar mills optimise siding rosters to increase profitability. Annals of Operations Research, 128, 235-249.

Hooker, J. N. (2010). Optimality conditions for distributive justice. International Transactions in Operational Research, 17(4), 485-505.

Hooker, J. N., \& Williams, H. P. (2012). Combining equity and utilitarianism in a mathematical programming model. Management Science, 58(9), 1682-1693.

Huang, M., Smilowitz, K., \& Balcik, B. (2012). Models for relief routing: Equity, efficiency and efficacy. Transportation Research Part E: Logistics and Transportation Review, $48(1), 2-18$.

Jahn, O., Möhring, R. H., Schulz, A. S., \& Stier-Moses, N. E. (2005). System-optimal routing of traffic flows with user constraints in networks with congestion. Operations Research, 53(4), 600-616.

Jang, W., Lim, H. H., Crowe, T. J., Raskin, G., \& Perkins, T. E. (2006). The Missouri Lottery optimizes its scheduling and routing to improve efficiency and balance. Interfaces, 36(4), 302-313.

Jeong, S. B., Kim, S., \& Lee, H. (2005). Data traffic scheduling algorithm for multiuser OFDM system with adaptive modulation considering fairness among users. Computers \& Operations Research, 32(7), 1723-1737.

Jia, H., Ordóñez, F., \& Dessouky, M. (2007). A modeling framework for facility location of medical services for large-scale emergencies. IIE Transactions, 39(1), 41-55.

Johnson, M. P. (2003). Single-period location models for subsidized housing: Tenantbased subsidies. Annals of Operations Research, 123, 105-124.

Johnson, M. P., Turcotte, D. A., \& Sullivan, F. M. (2010). What foreclosed homes should a municipality purchase to stabilize vulnerable neighborhoods? Networks and Spatial Economics, 10(3), 363-388.

Jozefowiez, N., Semet, F., \& Talbi, E.-G. (2008). Multi-objective vehicle routing problems. European Journal of Operational Research, 189(2), 293-309.

Karsu, Ö., \& Morton, A. (2014). Incorporating balance concerns in resource allocation decisions: A bi-criteria modelling approach. Omega, 44, 70-82.
Karsu, Ö., Morton, A., \& Argyris, N. (2012). Incorporating preference information in multicriteria problems with equity concerns. Technical Report London School of Economics and Political Science. LSEOR 13.136.

Kelly, F. P., Massoulié, L., \& Walton, N. S. (2009). Resource pooling in congested networks: Proportional fairness and product form. Queueing Systems, 63(1-4), 165-194.

Kelly, F. P., Maulloo, A. K., \& Tan, D. K. H. (1998). Rate control for communication networks: Shadow prices, proportional fairness and stability. The Journal of the Operational Research Society, 49(3), 237-252.

Kimbrel, T., Schieber, B., \& Sviridenko, M. (2006). Minimizing migrations in fair multiprocessor scheduling of persistent tasks. Journal of Scheduling, 9(4), 365-379.

Köppen, M. (2013). Relational optimization and its application: From bottleneck flow control to wireless channel allocation. Informatica, Lithuanian Academy of Sciences, 24(3), 413-433.

Köppen, M., Yoshida, K., Ohnishi, K., \& Tsuru, M. (2012). Meta-heuristic approach to proportional fairness. Evolutionary Intelligence, 5(4), 231-244.

Kostreva, M. M., \& Ogryczak, W. (1999). Linear optimization with multiple equitable criteria. RAIRO Operations Research, 33, 275-297.

Kostreva, M. M., Ogryczak, W., \& Wierzbicki, A. (2004). Equitable aggregations and multiple criteria analysis. European Journal of Operational Research, 158(2), 362-377.

Kotnyek, B., \& Richetta, O. (2006). Equitable models for the stochastic ground-holding problem under collaborative decision making. Transportation Science, 40(2), 133-146.

Kozanidis, G. (2009). Solving the linear multiple choice knapsack problem with two objectives: Profit and equity. Computational Optimization and Applications, 43(2), 261-294.

Kubiak, W. (2009). Proportional optimization and fairness. Springer.

Kunqi, G., Lixin, S., \& Shilou, J. (2007). Utility function based fair data scheduling algorithm for OFDM wireless network. Journal of Systems Engineering and Electronics, 18(4), 731-738.

Lee, C. Y., \& Cho, H. K. (2007). Discrete bandwidth allocation considering fairness and transmission load in multicast networks. Computers \& Operations Research, 34(3), 884-899.

Lee, C. Y., Moon, Y. P., \& Cho, Y. J. (2004). A lexicographically fair allocation of discrete bandwidth for multirate multicast traffics. Computers \& Operations Research, 31(14), 2349-2363.

Lejeune, M. A., \& Prasad, S. Y. (2013). Effectiveness-equity models for facility location problems on tree networks. NETWORKS, 62(4), 243-254.

Levinson, D. (2010). Equity effects of road pricing: A review. Transport Reviews, 30(1), 33-57.

Li, Y., Yang, M., Chen, Y., Dai, Q., \& Liang, L. (2013). Allocating a fixed cost based on data envelopment analysis and satisfaction degree. Omega, 41(1), 55-60.

Lo, H. K., \& Szeto, W. (2009). Time-dependent transport network design under cost-recovery. Transportation Research Part B: Methodological, 43(1), 142-158.

Lulli, G., \& Odoni, A. (2007). The European air traffic flow management problem. Transportation Science, 41(4), 431-443.

Luss, H. (2008). An equitable bandwidth allocation model for video-on-demand networks. Networks and Spatial Economics, 8(1), 23-41.

Luss, H. (2010). Equitable bandwidth allocation in content distribution networks. Naval Research Logistics, 57(3), 266-278.

Luss, H. (2012a). A distributed algorithm for equitable bandwidth allocation for content distribution in a tree network. Journal of the Operational Research Society, $63(4), 460-469$.

Luss, H. (2012b). Equitable resource allocation. John Wiley and Sons.

Maliszewski, P. J., Kuby, M. J., \& Horner, M. W. (2012). A comparison of multi-objective spatial dispersion models for managing critical assets in urban areas. Computers, Environment and Urban Systems, 36(4), 331-341.

Mandelbaum, A., Momčilović, P., \& Tseytlin, Y. (2012). On fair routing from emergency departments to hospital wards : QED queues with heterogeneous servers. Management Science, 58(7), 1273-1291.

Marín, A., Nickel, S., \& Velten, S. (2010). An extended covering model for flexible discrete and equity location problems. Mathematical Methods of Operations Research, 71(1), 125-163.

Marsh, M. T., \& Schilling, D. A. (1994). Equity measurement in facility location analysis A review and framework. European Journal of Operational Research, 74, 1-17.

Marshall, A., Olkin, I., \& Arnold, B. (2009). Inequalities: Theory of majorization and its applications. Springer series in statistics (2nd ed.). New York, USA: Springer.

Martin, S., Ouelhadj, D., Smet, P., Vanden Berghe, G., \& Özcan, E. (2013). Cooperative search for fair nurse rosters. Expert Systems with Applications, 40(16), 6674-6683.

Mclay, L. A., \& Mayorga, M. E. (2013). A dispatching model for server-to-customer systems that balances efficiency and equity. Manufacturing $\mathcal{E}$ Service Operations Management, 15(2), 205-220.

Medernach, E., \& Sanlaville, E. (2012). Fair resource allocation for different scenarios of demands. European Journal of Operational Research, 218(2), 339-350.

Melachrinoudis, E., \& Xanthopulos, Z. (2003). Semi-obnoxious single facility location in Euclidean space. Computers \& Operations Research, 30(14), 2191-2209.

Mestre, A. M., Oliveira, M. D., \& Barbosa-Póvoa, A. (2012). Organizing hospitals into networks: A hierarchical and multiservice model to define location, supply and referrals in planned hospital systems. OR Spectrum, 34(2), 319-348.

Miyagawa, M. (2009). Optimal hierarchical system of a grid road network. Annals of Operations Research, 172(1), 349-361.

Mladenovic, N., Labbe, M., \& Hansen, P. (2003). Solving the p-center problem with tabu search and variable neighborhood search. Networks, 42(1), 48-64.

Morell, A., Seco-Granados, G., \& Vázquez-Castro, M. A. (2008). Cross-layer design of dynamic bandwidth allocation in DVB-RCS. IEEE Systems Journal, 2(1), 62-73. 
López-de-los Mozos, M., Mesa, J. A., \& Puerto, J. (2008). A generalized model of equality measures in network location problems. Computers \& Operations Research, 35(3), 651-660.

López-de-los Mozos, M., Puerto, J., \& Rodríguez-Chía, A. M. (2013). Robust mean absolute deviation problems on networks with linear vertex weights. Networks, 61(1), 76-85.

Lopez-de-los mozos, M. C., \& Mesa, J. A. (2003). The sum of absolute differences on network: Algorithm and comparison with other equality measures. INFOR, 41(2), $195-210$.

Mukherjee, A., \& Hansen, M. (2007). A dynamic stochastic model for the single airport ground holding problem. Transportation Science, 41(4), 444-456.

Mulligan, G. F. (1991). Equality measures and facility location. The Journal of the RSAI, 70(4), 345-365

Mut, M., \& Wiecek, M. M. (2011). Generalized equitable preference in multiobjective programming. European Journal of Operational Research, 212(3), 535-551.

Nace, D., Doan, L. N., Klopfenstein, O., \& Bashllari, A. (2008). Max-min fairness in multi-commodity flows. Computers E' Operations Research, 35(2), 557-573.

Nace, D., \& Orlin, J. B. (2007). Lexicographically minimum and maximum load linear programming problems. Operations Research, 55(1), 182-187.

Ogryczak, W. (2007). Multicriteria models for fair resource allocation. Control and Cybernetics, 36(2), 303-332.

Ogryczak, W. (2009). Inequality measures and equitable locations. Annals of Operations Research, 167(1), 61-86.

Ogryczak, W., Luss, H., Pióro, M., Nace, D., \& Tomaszewski, A. (2014). Fair optimization and networks: A survey. Journal of Applied Mathematics, 2014, 612018. doi: $10.1155 / 2014 / 612018$

Ogryczak, W., Pioro, M., \& Tomaszewski, A. (2005). Telecommunications network design and max-min optimization problems. Journal of Telecommunications and Information Technology, 4, 43-56.

Ogryczak, W., \& Śliwiński, T. (2003). On solving linear programs with the ordered weighted averaging objective. European Journal of Operational Research, 148(1), 80-91.

Ogryczak, W., Wierzbicki, A., \& Milewski, M. (2008). A multi-criteria approach to fair and efficient bandwidth allocation. Omega, 36(3), 451-463.

Ohsawa, Y., Ozaki, N., \& Plastria, F. (2008). Equity-efficiency bicriteria location with squared Euclidean distances. Operations Research, 56(1), 79-87.

Ohsawa, Y., \& Tamura, K. (2003). Efficient location for a semi-obnoxious facility. Annals of Operations Research, 123, 173-188.

Pelegrín-Pelegrín, B., Dorta-González, P., \& Fernández-Hernández, P. (2011). Finding location equilibria for competing firms under delivered pricing. Journal of the Operational Research Society, 62(4), 729-741.

Perugia, A., Moccia, L., Cordeau, J.-F., \& Laporte, G. (2011). Designing a home-to-work bus service in a metropolitan area. Transportation Research Part B: Methodological, 45(10), 1710-1726.

Prokopyev, O. A., Kong, N., \& Martinez-Torres, D. L. (2009). The equitable dispersion problem. European Journal of Operational Research, 197(1), 59-67.

Ramos, T. R. P., \& Oliveira, R. C. (2011). Delimitation of service areas in reverse logistics networks with multiple depots. Journal of the Operational Research Society, 62(7), 1198-1210.

Rawls, J. (1971). A theory of justice. Cambridge, MA: Harvard University Press.

Ray, D. (1998). Development economics. Princeton University Press.

Rothschild, M., \& Stiglitz, J. H. (1973). Some further results on the measurement of inequality. Journal of Economic Theory, 6(2), 188-204.

Roy, B. (1971). Problems and methods with multiple objective functions. Mathematical Programming, 1(1), 239-266.

Ryan, S. M., \& Vorasayan, J. (2005). Allocating work in process in a multiple-product CONWIP system with lost sales. International Journal of Production Research, 43(2), 223-246.

Salles, R. M., \& Barria, J. A. (2008). Lexicographic maximin optimisation for fair bandwidth allocation in computer networks. European Journal of Operational Research, 185(2), 778-794.

Sandmann, W. (2013). Quantitative fairness for assessing perceived service quality in queues. Operational Research International Journal, 13(2), 153-186.

Sen, A. (1973). On economic inequality. Oxford: Clarendon Press.

Sherali, H. D., Hill, J. M., McCrea, M. V., \& Trani, A. A. (2011). Integrating slot exchange, safety, capacity, and equity mechanisms within an airspace flow program. Transportation Science, 45(2), 271-284.

Sherali, H. D., Staats, R. W., \& Trani, A. A. (2003). An airspace planning and collaborative decision-making model: Part I probabilistic conflicts, workload, and equity considerations. Transportation Science, 37(4), 434-456.

Sherali, H. D., Staats, R. W., \& Trani, A. A. (2006). An airspace-planning and collaborative decision-making model: Part II cost model, data considerations, and computations. Transportation Science, 40(2), 147-164.

Shorrocks, A. (1983). Ranking income distributions. Economica, 50, 3-17.
Smith, D. L., Nauss, R. M., Mattfeld, D. C., Li, J., Ehmke, J. F., \& Reindl, M. (2011). Scheduling operations at system choke points with sequence-dependent delays and processing times. Transportation Research Part E: Logistics and Transportation Review, 47(5), 669-680.

Smith, H. K., Harper, P. R., \& Potts, C. N. (2013). Bicriteria efficiency/equity hierarchical location models for public service application. Journal of the Operational Research Society, 64(4), 500-512.

Smith, H. K., Harper, P. R., Potts, C. N., \& Thyle, A. (2009). Planning sustainable community health schemes in rural areas of developing countries. European Journal of Operational Research, 193(3), 768-777.

Stolletz, R., \& Brunner, J. O. (2012). Fair optimization of fortnightly physician schedules with flexible shifts. European Journal of Operational Research, 219(3), 622-629.

Suzuki, A., \& Drezner, Z. (2009). The minimum equitable radius location problem with continuous demand. European Journal of Operational Research, 195(1), 17-30.

Swaminathan, J. M. (2003). Decision support for allocating scarce drugs. Interfaces, 33(2), 1-11.

Swaminathan, J. M., Ashe, M., Duke, K., Maslin, L., \& Wilde, L. (2004). Distributing scarce drugs for the Medpin program. Interfaces, 34(5), 353-358.

Szeto, W., \& Lo, H. K. (2006). Transportation network improvement and tolling strategies: The issue of intergeneration equity. Transportation Research Part A: Policy and Practice, 40(3), 227-243.

Tomaszewski, A. (2005). A polynomial algorithm for solving a general max-min fairness problem. European Transactions on Telecommunications, 16(3), 233-240.

Tsai, C.-C., \& Li, S. H. (2009). A two-stage modeling with genetic algorithms for the nurse scheduling problem. Expert Systems with Applications, 36(5), 9506-9512

Turkcan, A., Zeng, B., Muthuraman, K., \& Lawley, M. (2011). Sequential clinical scheduling with service criteria. European Journal of Operational Research, 214(3), 780-795.

Tzeng, G. H., Cheng, H., \& Huang, T. D. (2007). Multi-objective optimal planning for designing relief delivery systems. Transportation Research Part E: Logistics and Transportation Review, 43(6), 673-686.

Udías, A., Ríos Insua, D., Cano, J., \& Fellag, H. (2012). Cost-efficient equitable water distribution in Algeria: A bicriteria fair division problem with network constraints. International Transactions in Operational Research, 19(5), 675-693.

van 't Hof, P., Post, G., \& Briskorn, D. (2010). Constructing fair round robin tournaments with a minimum number of breaks. Operations Research Letters, 38(6), 592-596.

Verloop, I. M., Ayesta, U., \& Borst, S. (2010). Monotonicity properties for multi-class queueing systems. Discrete Event Dynamic Systems, 20, 473-509.

Vitoriano, B., Ortuño, M. T., Tirado, G., \& Montero, J. (2011). A multi-criteria optimization model for humanitarian aid distribution. Journal of Global Optimization, 51(2), 189-208.

Vossen, T., \& Ball, M. (2006). Optimization and mediated bartering models for ground delay programs. Naval Research Logistics, 53(1), 75-90.

Walton, N. S. (2011). Insensitive, maximum stable allocations converge to proportional fairness. Queueing Systems, 68(1), 51-60

Wang, L., Fang, L., \& Hipel, K. W. (2007). On achieving fairness in the allocation of scarce resources: Measurable principles and multiple objective optimization approaches. IEEE Systems Journal, 1(1), 17-28.

Wang, L., Fang, L., \& Hipel, K. W. (2008). Basin-wide cooperative water resources allocation. European Journal of Operational Research, 190(3), 798-817.

Ward, A. R., \& Armony, M. (2013). Blind Fair Routing in Large-Scale Service Systems with Heterogeneous Customers and Servers. Operations Research, 61(1), 228-243.

Wu, D., Yin, Y., Lawphongpanich, S., \& Yang, H. (2012). Design of more equitable congestion pricing and tradable credit schemes for multimodal transportation networks. Transportation Research Part B: Methodological, 46(9), 1273-1287.

Yang, M., Allen, T. T., Fry, M. J., \& Kelton, W. D. (2013). The call for equity: simulation optimization models to minimize the range of waiting times. IIE Transactions, 45(7), 781-795.

Young, P. H. (1994). Equity in theory and practice (1st ed.). Princeton: Princeton University Press.

Zanjirani Farahani, R., \& Hekmatfar, M. E. (2009). Facility location: Concepts, models, algorithms and case studies. Contributions to management science. Heidelberg: Physica-Verlag.

Zhang, H., \& Shen, W. (2010). Access control policies without inside queues: Their properties and public policy implications. Transportation Research Part B: Methodological, 44(8-9), 1132-1147.

Zhang, J., \& Ansari, N. (2010). An Application-Oriented Fair Resource Allocation Scheme for EPON. IEEE Systems Journal, 4(4), 424-431.

Zukerman, M., Mammadov, M., Tan, L., Ouveysi, I., \& Andrew, L. L. (2008). To be fair or efficient or a bit of both. Computers \& Operations Research, 35(12), 3787-3806. 